\title{
Asymptotic Properties and Hausdorff Dimensions of Fractional Brownian Sheets
}

\author{
Antoine Ayache and Yimin Xiao \\ Communicated by Christian Houdré
}

\begin{abstract}
Let $B^{H}=\left\{B^{H}(t), t \in \mathbb{R}^{N}\right\}$ be an $(N, d)$-fractional Brownian sheet with index $H=\left(H_{1}, \ldots, H_{N}\right) \in(0,1)^{N}$. The uniform and local asymptotic properties of $B^{H}$ are proved by using wavelet methods. The Hausdorff and packing dimensions of the range $B^{H}\left([0,1]^{N}\right)$, the graph $\mathrm{Gr} B^{H}\left([0,1]^{N}\right)$ and the level set are determined.
\end{abstract}

\section{Introduction}

For a given vector $H=\left(H_{1}, \ldots, H_{N}\right)\left(0<H_{j}<1\right.$ for $\left.j=1, \ldots, N\right)$, a one-dimensional fractional Brownian sheet $B_{0}^{H}=\left\{B_{0}^{H}(t), t \in \mathbb{R}^{N}\right\}$ with Hurst index $H$ is a real-valued, centered Gaussian random field with covariance function given by

$$
\mathbb{E}\left[B_{0}^{H}(s) B_{0}^{H}(t)\right]=\prod_{j=1}^{N} \frac{1}{2}\left(\left|s_{j}\right|^{2 H_{j}}+\left|t_{j}\right|^{2 H_{j}}-\left|s_{j}-t_{j}\right|^{2 H_{j}}\right), \quad s, t \in \mathbb{R}^{N} .
$$

It follows from (1.1) that $B_{0}^{H}$ is an anisotropic Gaussian random field and $B_{0}^{H}(t)=0$ a.s. for every $t \in \mathbb{R}^{N}$ with at least one zero coordinate. Moreover, $B_{0}^{H}$ has the following invariance properties:

(i) $B_{0}^{H}$ is self-similar in the sense that for all constants $c>0$,

$$
\left\{B_{0}^{H}(c t), t \in \mathbb{R}^{N}\right\} \stackrel{d}{=}\left\{c^{\sum_{j=1}^{N} H_{j}} B_{0}^{H}(t), t \in \mathbb{R}^{N}\right\} .
$$

Math Subject Classifications. 60G15, 60G17, 42C40, 28A80.

Keywords and Phrases. Fractional Brownian sheet, wavelet representation, modulus of continuity, Hausdorff dimension, packing dimension, range, graph, level set.

Acknowledgements and Notes. Second author was partially supported by NSF grant DMS-0103939. 
(ii) Let $W^{H}=\left\{W^{H}(t), t \in \mathbb{R}^{N}\right\}$ be the Gaussian random field defined by

$$
W^{H}(t)=\left\{\begin{array}{lc}
\prod_{j=1}^{N}\left|t_{j}\right|^{2 H_{j}} B_{0}^{H}\left(t_{1}^{-1}, \ldots, t_{N}^{-1}\right) & \text { if } t_{j} \neq 0 \text { for all } j \\
0 & \text { otherwise }
\end{array}\right.
$$

Then, $W^{H} \stackrel{d}{=} B_{0}^{H}$.

In the above, $\stackrel{d}{=}$ means equality in the finite dimensional distributions.

Fractional Brownian sheet has the following stochastic integral representation

$$
B_{0}^{H}(t)=\kappa_{H}^{-1} \int_{-\infty}^{t_{1}} \cdots \int_{-\infty}^{t_{N}} g(t, s) W(d s),
$$

where $W=\left\{W(s), s \in \mathbb{R}^{N}\right\}$ is the standard real-valued Brownian sheet and

$$
g(t, s)=\prod_{j=1}^{N}\left[\left(\left(t_{j}-s_{j}\right)_{+}\right)^{H_{j}-1 / 2}-\left(\left(-s_{j}\right)_{+}\right)^{H_{j}-1 / 2}\right]
$$

with $s_{+}=\max \{s, 0\}$, and where $\kappa_{H}$ is the normalizing constant given by

$$
\kappa_{H}^{2}=\int_{-\infty}^{1} \cdots \int_{-\infty}^{1}\left[\prod_{j=1}^{N} g^{2}(\langle 1\rangle, s)\right]^{2} d s
$$

so that $\mathbb{E}\left[\left(B_{0}^{H}(t)\right)^{2}\right]=\prod_{j=1}^{N}\left|t_{j}\right|^{2 H_{j}}$ for all $t \in \mathbb{R}^{N}$. Here $\langle 1\rangle=(1,1, \ldots, 1) \in \mathbb{R}^{N}$.

Let $B_{1}^{H}, \ldots, B_{d}^{H}$ be $d$ independent copies of $B_{0}^{H}$. Then the $(N, d)$-fractional Brownian sheet with Hurst index $H=\left(H_{1}, \ldots, H_{N}\right)$ is the Gaussian random field $B^{H}=\left\{B^{H}(t)\right.$ : $\left.t \in \mathbb{R}^{N}\right\}$ with values in $\mathbb{R}^{d}$ defined by

$$
B^{H}(t)=\left(B_{1}^{H}(t), \ldots, B_{d}^{H}(t)\right), \quad t \in \mathbb{R}^{N} .
$$

Note that, if $N=1$, then $B^{H}$ is a fractional Brownian motion in $\mathbb{R}^{d}$ with Hurst index $H_{1} \in(0,1)$; if $N>1$ and $H_{1}=\cdots=H_{N}=1 / 2$, then $B^{H}$ is the $(N, d)$-Brownian sheet. Hence, $B^{H}$ can be regarded as a natural generalization of one parameter fractional Brownian motion in $\mathbb{R}^{d}$ to $(N, d)$ Gaussian random fields, as well as a generalization of the Brownian sheet. Another well known generalization is the multiparameter fractional Brownian motion $X=\left\{X(t), t \in \mathbb{R}^{N}\right\}$, which is a centered Gaussian random field with covariance function

$$
\mathbb{E}\left[X_{i}(s) X_{j}(t)\right]=\frac{1}{2} \delta_{i j}\left(|s|^{2 H_{1}}+|t|^{2 H_{1}}-|s-t|^{2 H_{1}}\right), \quad \forall s, t \in \mathbb{R}^{N},
$$

where $0<H_{1}<1$ is a constant and $\delta_{i j}=1$, if $i=j$ and 0 , if $i \neq j$, and where $|\cdot|$ denotes the Euclidean norm in $\mathbb{R}^{N}$.

Fractional Brownian sheets arise naturally in many areas, including in stochastic partial differential equations (cf. Øksendal and Zhang [26], Hu, Øksendal and Zhang [16]) and in studies of most visited sites of symmetric Markov processes (cf. Eisenbaum and Khoshnevisan [14]).

Recently, many authors have studied various properties of the fractional Brownian sheets. For example, Dunker [11] has studied the small ball probability of an $(N, 1)$ fractional Brownian sheet. For the special class of fractional Brownian sheets such that 
there is a unique minimum among $H_{1}, \ldots, H_{N}$, Mason and Shi [25] have obtained the exact rate for the small ball probability and have computed the Hausdorff dimension of some exceptional sets related to the oscillation of their sample paths. Belinski and Linde [6] give a different proof of the small ball probability result of Mason and Shi [25] and have also obtained a sharp estimate for the small ball probability in the case of $N=2$ and $H_{1}=H_{2}$. More generally, by using different kinds of $s$-numbers Kühn and Linde [21] have determined the rate, up to a logarithmic factor, for the small ball probability and the optimality of series representations for the fractional Brownian sheet $B^{H}$ with an arbitrary index $H=\left(H_{1}, \ldots, H_{N}\right) \in(0,1)^{N}$. Ayache and Taqqu [4] have derived optimal wavelet series expansions for the fractional Brownian sheet $B^{H}$; see also Dzhaparidze and van Zanten [12] for other optimal infinite series expansions. Xiao and Zhang [36] have proved a sufficient condition for the joint continuity of the local times of an $(N, d)$-fractional Brownian sheet $B^{H}$. Kamont [18] and Ayache [3] have studied the box-dimension and the Hausdorff dimension of the graph set of an $(N, 1)$-fractional Brownian sheet $B^{H}$ using wavelet methods.

The main objective of this article is to further investigate the asymptotic and fractal properties of the $(N, d)$-fractional Brownian sheet $B^{H}$. We are particularly interested in describing the anisotropic nature of $B^{H}$ in terms of the Hurst index $H=\left(H_{1}, \ldots, H_{N}\right) \in$ $(0,1)^{N}$. We should mention that several authors have been interested in applying anisotropic Gaussian random fields to stochastic modelling; see, for example, Bonami and Estrade [9] for bone structure modelling, and Benson et al. [7] for modelling aquifer structure in hydrology. We hope that the results and techniques in this article will be helpful for studying more general anisotropic Gaussian random fields.

The rest of this article is organized as follows. In Section 2, we study the uniform and local modulus of continuity and the law of the iterated logarithm of an $(N, 1)$-fractional Brownian sheet. Many authors have studied the asymptotic behavior of the sample functions of Gaussian random fields (see, e.g., Albin [2] and Kôno [20] and the reference therein). Our approach is different from those in the aforementioned references and relies on the wavelet expansion of $B^{H}$ in terms of a Lemarié-Meyer wavelet basis for $L^{2}(\mathbb{R})$. We remark that, even though the methods of Albin [2] and Kôno [20] based on metric entropy may be modified to prove our Theorems 1 and 2 below, our wavelet-based approach has certain advantages. In particular, it allows us to prove that, with probability 1 , the sample function $B^{H}(t)$ does not satisfy any local Hölder conditions with respect to the function $\sigma^{1+\varepsilon}(t+h, t)$, for any $\varepsilon>0$, where $\sigma^{2}(t+h, t)=\mathbb{E}\left[\left(B^{H}(t+h)-B^{H}(t)\right)^{2}\right]$; see Theorem 3. This implies that $B^{H}(t)$ is nowhere differentiable on $\mathbb{R}^{N}$. Theorem 3 is more difficult to establish directly due to the complex dependence structure of $B^{H}$. It is worthwhile to mention that another way of proving the non-differentiability of $B^{H}$ is by investigating the regularity of the local times of $B^{H}$. This approach relies on solving Problem 4.12 in Xiao and Zhang [36] and requires totally different techniques. We will deal with it elsewhere.

In Section 3, we determine the Hausdorff and packing dimensions of the range $B^{H}\left([0,1]^{N}\right)$, the graph $\operatorname{Gr} B^{H}\left([0,1]^{N}\right)$ and the level set $L_{x}=\left\{t \in(0, \infty)^{N}: B^{H}(t)=x\right\}$. Our method for determining the Hausdorff dimension of $\operatorname{Gr} B^{H}\left([0,1]^{N}\right)$ is different from that in Ayache [3], and is more reminiscent to the arguments in Xiao [31]. Our results suggest that, due to the anisotropy of $B^{H}$ in $t$, the sample paths of an $(N, d)$-fractional Brownian sheet $B^{H}$ are more irregular than those of the Brownian sheet or an $(N, d)$ fractional Brownian motion.

Finally, we introduce some notation. Throughout this article, the underlying parameter space is $\mathbb{R}^{N}$, or $\mathbb{R}_{+}^{N}=[0, \infty)^{N}$. A typical parameter, $t \in \mathbb{R}^{N}$ is written as 
$t=\left(t_{1}, \ldots, t_{N}\right)$, or occasionally, as $\langle c\rangle$, if $t_{1}=\cdots=t_{N}=c$. For any $s, t \in \mathbb{R}^{N}$ such that $s_{j}<t_{j}(j=1, \ldots, N)$, we define the closed interval (or rectangle) $[s, t]=\prod_{j=1}^{N}\left[s_{j}, t_{j}\right]$. We use $\langle\cdot, \cdot\rangle$ and $|\cdot|$ to denote the ordinary scalar product and the Euclidean norm in $\mathbb{R}^{m}$, respectively, no matter the value of the integer $m$.

We will use $c$ to denote an unspecified positive and finite constant which may not be the same in each occurrence. More specific constants in section $i$ are numbered as $c_{i, 1}, c_{i, 2}, \ldots$, and so on.

\section{Modulus of Continuity and Asymptotic Properties}

In this section, we investigate the uniform and local modulus of continuity, nowhere differentiability and the laws of the iterated logarithm of the fractional Brownian sheet $B^{H}=\left\{B^{H}(t), t \in \mathbb{R}^{N}\right\}$ with index $H=\left(H_{1}, H_{2}, \ldots, H_{N}\right), 0<H_{j}<1$. Our approach is based on the wavelet expansion of $B^{H}$.

For simplicity, we will suppose, in this section, that $B^{H}$ is real-valued (i.e., $d=1$ ), and we will use $A, A_{1}, A_{2}, \ldots$ to denote positive random variables.

Let us first state the main results.

Theorem 1. There exist a random variable $A_{1}=A_{1}(\omega)>0$ of finite moments of any order and an event $\Omega_{1}^{*}$ of probability 1 such that for any $\omega \in \Omega_{1}^{*}$,

$$
\sup _{s, t \in[0,1]^{N}} \frac{\left|B^{H}(s, \omega)-B^{H}(t, \omega)\right|}{\sum_{j=1}^{N}\left|s_{j}-t_{j}\right|^{H_{j}} \sqrt{\log \left(3+\left|s_{j}-t_{j}\right|^{-1}\right)}} \leq A_{1}(\omega) .
$$

\section{Remark 1.}

- Up to a constant, the inequality in Theorem 1 is sharp. When $H_{1}=\cdots=H_{N}=\frac{1}{2}$ it agrees with the corresponding result for the Brownian sheet due to Orey and Pruitt [28],

- Theorem 1 remains valid when $[0,1]^{N}$ is replaced by any compact rectangle of $\mathbb{R}^{N}$.

- The event $\Omega_{1}^{*}$ will be specified in the proof of Theorem 1 .

- As we mentioned in the introduction, Theorem 1 can also be proven using the method of proving Theorem 1 in Kôno [20]. The proof we give below is based on the wavelet representation of $B^{H}$.

Next we give an upper bound for the asymptotic behavior of the fractional Brownian sheet $B^{H}(t)$ as $|t| \rightarrow \infty$. Recall that, by the law of the iterated logarithm (see, for example, Orey [27]), the ordinary fractional Brownian motion $\left\{B_{H}(t), t \in \mathbb{R}\right\}$ with Hurst index $H \in(0,1)$ satisfies with probability 1 ,

$$
\left|B_{H}(t)\right| \leq A(1+|t|)^{H} \sqrt{\log \log (3+|t|)}, \quad \forall t \in \mathbb{R},
$$

where $A$ is a positive random variable. This result can be extended to the fractional Brownian sheet as follows. 
Theorem 2. Let $B^{H}=\left\{B^{H}(t), t \in \mathbb{R}^{N}\right\}$ be a fractional Brownian sheet with index $H=\left(H_{1}, \ldots, H_{N}\right)$ and let $\Omega_{1}^{*}$ be the event of probability 1 in Theorem 1. Then, there is a random variable $A_{2}>0$ of finite moments of any order such that for all $\omega \in \Omega_{1}^{*}$,

$$
\sup _{t \in \mathbb{R}^{N}} \frac{\left|B^{H}(t, \omega)\right|}{\prod_{j=1}^{N}\left(1+\left|t_{j}\right|\right)^{H_{j}} \sqrt{\log \log \left(3+\left|t_{j}\right|\right)}} \leq A_{2}(\omega) .
$$

Note that (2.2) is more concerned with the global property of $B^{H}(t)$ and it does not catch the local asymptotic behavior of $B^{H}(t)$ near $t=0$. To study the asymptotic properties of $B^{H}$ at a fixed point $t_{0} \in \mathbb{R}^{N}$, we consider

$$
\sigma^{2}(s, t)=\mathbb{E}\left[\left(B^{H}(t)-B^{H}(s)\right)^{2}\right], \quad \forall s, t \in \mathbb{R}^{N} .
$$

It follows from the proof of Lemma 8 below that for any closed interval $I=[a, b]$, there is a finite constant $c_{2,1}>0$ such that for all $s, t \in I$,

$$
\sigma(t, s) \leq c_{2,1} \sum_{j=1}^{N}\left|t_{j}-s_{j}\right|^{H_{j}} .
$$

Hence, one can apply the metric entropy method to prove a law of the iterated logarithm for $B^{H}$. The following is a consequence of the proof of Theorem 2 in Kôno [20].

Proposition 1. There exists a positive and finite constant $c_{2,2}$ such that for every $t_{0} \in I$, with probability 1

$$
\limsup _{h \rightarrow 0} \frac{\left|B^{H}\left(t_{0}+h\right)-B^{H}\left(t_{0}\right)\right|}{\sigma\left(t_{0}, t_{0}+h\right) \sqrt{\log \log \frac{1}{|h|}}} \leq c_{2,2} .
$$

The next result is a partial inverse of (2.5) and implies that, for every $\varepsilon>0$, the sample function $B^{H}(t)$ does not satisfy $\sigma^{1+\varepsilon}$-Hölder condition at any point, where $\sigma(t, s)$ is defined in (2.3).

Theorem 3. Let $B^{H}=\left\{B^{H}(t), t \in \mathbb{R}^{N}\right\}$ be a real-valued fractional Brownian sheet with index $H=\left(H_{1}, \ldots, H_{N}\right)$. Then, there is an event $\Omega_{2}^{*}$ of probability 1 such that for all $\epsilon>0, t \in(0,1]^{N}$ and all $\omega \in \Omega_{2}^{*}$,

$$
\limsup _{|h| \rightarrow 0} \frac{\left|B^{H}(t+h, \omega)-B^{H}(t, \omega)\right|}{\sigma(t+h, t)^{1+\epsilon}}=\infty .
$$

In order to prove Theorems 1,2, and 3, we will use the wavelet representation of the fractional Brownian sheet introduced in Ayache [3]. To this end, we need to introduce the following notation.

- $\left\{2^{J / 2} \psi\left(2^{J} x-K\right),(J, K) \in \mathbb{Z} \times \mathbb{Z}\right\}$ will be a Lemarié-Meyer wavelet basis for $L^{2}(\mathbb{R})$ (see, for instance, Lemarié and Meyer [23], or Meyer [24]). Recall that such orthonormal bases satisfy the following properties:

(a) $\quad \psi$ and its Fourier transform $\widehat{\psi}$ belong to the Schwartz class $\mathcal{S}(\mathbb{R})$, namely the space of all infinitely differentiable functions $u$ which verify for all integers $n \geq 0$ and $m \geq 0$,

$$
\lim _{|t| \rightarrow \infty} t^{m}\left(\frac{d}{d t}\right)^{n} u(t)=0 .
$$


Recall that the Fourier transform of a function $f \in L^{1}(\mathbb{R})$ is defined as $\widehat{f}(\xi)=\int_{\mathbb{R}} e^{-i \xi x} f(x) d x$ for all $\xi \in \mathbb{R}$.

(b) $\widehat{\psi}$ is even, compactly supported and vanishes in a neighborhood of the origin. More precisely, the support of $\widehat{\psi}$ is contained in the domain $\left\{\xi: \frac{2 \pi}{3} \leq|\xi| \leq\right.$ $\left.\frac{8 \pi}{3}\right\}$.

- For any $\alpha \in(0,1)$, the functions $\psi^{\alpha}$ and $\psi^{-\alpha}$ will denote, respectively the fractional primitive of order $\alpha+\frac{1}{2}$ and the fractional derivative of order $\alpha+\frac{1}{2}$ of the mother wavelet $\psi$, which are defined for all $x \in \mathbb{R}$ as

$$
\psi^{\alpha}(x)=\frac{1}{2 \pi} \int_{\mathbb{R}} e^{i x \xi} \frac{\widehat{\psi}(\xi)}{|\xi|^{\alpha+1 / 2}} d \xi \text { and } \psi^{-\alpha}(x)=\frac{1}{2 \pi} \int_{\mathbb{R}} e^{i x \xi}|\xi|^{\alpha+1 / 2} \widehat{\psi}(\xi) d \xi .
$$

In view of properties (a) and (b) of the Lemarié-Meyer wavelets, these definitions make sense and $\psi^{\alpha}$ and $\psi^{-\alpha}$ are real-valued and belong to the Schwartz class $\mathcal{S}(\mathbb{R})$. Moreover, we have for every $\xi \in \mathbb{R}$,

$$
\widehat{\psi^{\alpha}}(\xi)=\frac{\widehat{\psi}(\xi)}{|\xi|^{\alpha+1 / 2}} \quad \text { and } \quad \widehat{\psi^{-\alpha}}(\xi)=|\xi|^{\alpha+1 / 2} \widehat{\psi}(\xi) \text {. }
$$

- For any $H=\left(H_{1}, \ldots, H_{N}\right) \in(0,1)^{N}$ and for all $j=\left(j_{1}, \ldots, j_{N}\right) \in \mathbb{Z}^{N}$, $k=\left(k_{1}, \ldots, k_{N}\right) \in \mathbb{Z}^{N}$ and $t=\left(t_{1}, \ldots, t_{N}\right) \in \mathbb{R}^{N}$, we set

$$
\widetilde{\Psi}_{j, k}^{(H)}(t)=\prod_{l=1}^{N} \widetilde{\psi}_{j_{l}, k_{l}}^{H_{l}}\left(t_{l}\right),
$$

where for any $\alpha \in(0,1), x \in \mathbb{R}$ and any $(J, K) \in \mathbb{Z}^{2}$,

$$
\widetilde{\psi}_{J, K}^{\alpha}(x)=\psi^{\alpha}\left(2^{J} x-K\right)-\psi^{\alpha}(-K) .
$$

We are now in a position to give the wavelet representation of fractional Brownian sheet.

Proposition 2 (Ayache [3]). There is a sequence $\left\{\epsilon_{j, k},(j, k) \in \mathbb{Z}^{N} \times \mathbb{Z}^{N}\right\}$ of independent $\mathcal{N}(0,1)$ Gaussian random variables such that for any $H=\left(H_{1}, \ldots, H_{N}\right) \in(0,1)^{N}$, the fractional Brownian sheet $B^{H}=\left\{B^{H}(t), t \in \mathbb{R}^{N}\right\}$ can be represented (up to a multiplicative constant that only depends on $H$ ) as:

$$
B^{H}(t, \omega)=\sum_{(j, k) \in \mathbb{Z}^{N} \times \mathbb{Z}^{N}} 2^{-\langle j, H\rangle} \epsilon_{j, k}(\omega) \widetilde{\Psi}_{j, k}^{(H)}(t), \quad \forall t \in \mathbb{R}^{N},
$$

where $\langle j, H\rangle=\sum_{l=1}^{N} j_{l} H_{l}$ and where the series in (2.11) is, for each $t$, convergent in the $L^{2}(\Omega)$-norm ( $\Omega$ being the underlying probability space).

In fact, the series (2.11) is also convergent in a much stronger sense. More precisely, we have the following.

Proposition 3. For almost all $\omega \in \Omega$, the series (2.11) is uniformly convergent in t, on any compact subset of $\mathbb{R}^{N}$.

Proposition 3 can be obtained by using the same method as that of Ayache and Taqqu [4]. But since we are not interested in determining the rate of convergence of the series (2.11), this proposition can also be proved more simply as follows. 
Proof. For simplicity we will only prove that, with probability 1 , the series (2.11) converges uniformly in $t \in[0,1]^{N}$. For any $n \in \mathbb{N}$ and $t \in \mathbb{R}^{N}$, let

$$
B_{n}^{H}(t)=\sum_{(j, k) \in I_{n}} 2^{-\langle j, H\rangle} \epsilon_{j, k} \widetilde{\Psi}_{j, k}^{(H)}(t),
$$

where $I_{n}=\left\{(j, k) \in \mathbb{Z}^{N} \times \mathbb{Z}^{N}:\left|j_{l}\right| \leq n\right.$ and $\left|k_{l}\right| \leq n$ for all $\left.1 \leq l \leq N\right\}$.

Since the functions $\widetilde{\Psi}_{j, k}^{(H)}$ are continuous and the random variables $\epsilon_{j, k}$ are symmetric and independent, the Itô-Nisio Theorem (see Theorem 2.1.1 in Kwapień and Woyczyński [22]) implies that, for proving the uniform convergence of series $(2.11)$ on $[0,1]^{N}$, it is sufficient to show that the sequence $\left\{B_{n}^{H}(t), t \in[0,1]^{N}\right\}_{n \in \mathbb{N}}$ is weakly relatively compact in $C\left([0,1]^{N}\right)$, the space of continuous functions on $[0,1]^{N}$ equipped with the usual topology of uniform convergence. Observe that (2.11), (2.3), and (2.4) imply that there is a constant $c_{2,3}>0$ such that for all $n \geq 1$ and all $t, t^{\prime} \in[0,1]^{N}$,

$$
\mathbb{E}\left[\left(B_{n}^{H}(t)-B_{n}^{H}\left(t^{\prime}\right)\right)^{2}\right] \leq c_{2,3}\left|t-t^{\prime}\right|^{2 \underline{H}},
$$

where $\underline{H}=\min \left\{H_{1}, \ldots, H_{N}\right\}$. Since (2.13) and Theorem 12.3 in Billingsley [8] entail the weak relative compactness of the sequence $\left\{B_{n}^{H}(t), t \in[0,1]^{N}\right\}_{n \in \mathbb{N}}$ in $C\left([0,1]^{N}\right)$, this finishes the proof of Proposition 3.

The proof of Theorem 1 mainly relies on the following two technical lemmas. The proof of Lemma 1 is similar to that of Lemma 4 of Ayache [3], or Lemma 2 of Ayache and Taqqu [4]. Hence, it is omitted.

Lemma 1. Let $\left\{\epsilon_{m}, m \in \mathbb{Z}^{N}\right\}$ be a sequence of $\mathcal{N}(0,1)$ Gaussian random variables. Then, there is a random variable $A_{3}>0$, of finite moments of any order such that for almost all $\omega \in \Omega$ and for all $m=\left(m_{1}, \ldots, m_{N}\right) \in \mathbb{Z}^{N}$,

$$
\left|\epsilon_{m}(\omega)\right| \leq A_{3}(\omega) \sqrt{\log \left(3+\sum_{i=1}^{N}\left|m_{i}\right|\right)} .
$$

We will also make use of the following elementary inequality: There is a constant $c>0$ such that for all $\left(m_{1}, \ldots, m_{N}\right) \in \mathbb{Z}^{N}$,

$$
\sqrt{\log \left(3+\sum_{i=1}^{N}\left|m_{i}\right|\right)} \leq c \prod_{i=1}^{N} \sqrt{\log \left(3+\left|m_{i}\right|\right)} .
$$

Lemma 2. For any $\alpha \in(0,1)$, define the functions

$$
S_{\alpha}(x, y)=\sum_{(J, K) \in \mathbb{Z}^{2}} 2^{-J \alpha}\left|\psi^{\alpha}\left(2^{J} x-K\right)-\psi^{\alpha}\left(2^{J} y-K\right)\right| \sqrt{\log (3+|J|+|K|)}
$$

and

$$
T_{\alpha}(x)=\sum_{(J, K) \in \mathbb{Z}^{2}} 2^{-J \alpha}\left|\widetilde{\psi}_{J, K}^{\alpha}(x)\right| \sqrt{\log (3+|J|+|K|)} .
$$

Then there is a constant $c_{2,4}>0$ such that for all $x, y \in[0,1]$, one has

$$
S_{\alpha}(x, y) \leq c_{2,4}|x-y|^{\alpha} \sqrt{\log \left(3+|x-y|^{-1}\right)}
$$


and

$$
T_{\alpha}(x) \leq c_{2,4} .
$$

Proof. First we prove (2.18). Since the function $\psi^{\alpha}$ belongs to the Schwartz class $\mathcal{S}(\mathbb{R})$, its derivative of any order $n \geq 0$, satisfies

$$
\left|\left(\frac{d}{d x}\right)^{n} \psi^{\alpha}(x)\right| \leq c_{2,5}(3+|x|)^{-2}, \quad \forall x \in \mathbb{R},
$$

where $c_{2,5}>0$ is a constant that only depends on $n$. For any $x, y \in[0,1]$, satisfying $x \neq y$, there is a unique integer $J_{0} \geq 0$ such that

$$
2^{-J_{0}-1}<|x-y| \leq 2^{-J_{0}} .
$$

We decompose $S_{\alpha}(x, y)$ into the following 3 parts:

$$
\begin{aligned}
& S_{\alpha, 1}(x, y)=\sum_{J=-\infty} \sum_{K=-\infty}^{\infty} 2^{-J \alpha}\left|\psi^{\alpha}\left(2^{J} x-K\right)-\psi^{\alpha}\left(2^{J} y-K\right)\right| \sqrt{\log (3+|J|+|K|)}, \\
& S_{\alpha, 2}(x, y)=\sum_{J=0}^{J_{0}} \sum_{K=-\infty}^{\infty} 2^{-J \alpha}\left|\psi^{\alpha}\left(2^{J} x-K\right)-\psi^{\alpha}\left(2^{J} y-K\right)\right| \sqrt{\log (3+|J|+|K|)}, \\
& S_{\alpha, 3}(x, y)=\sum_{J=J_{0}+1}^{\infty} \sum_{K=-\infty}^{\infty} 2^{-J \alpha}\left|\psi^{\alpha}\left(2^{J} x-K\right)-\psi^{\alpha}\left(2^{J} y-K\right)\right| \sqrt{\log (3+|J|+|K|)}
\end{aligned}
$$

and derive upper bounds for $S_{\alpha, 1}(x, y), S_{\alpha, 2}(x, y)$ and $S_{\alpha, 3}(x, y)$ separately.

Without loss of generality, we will assume $x<y$. It follows from the Mean-Value Theorem that for any integers $-\infty<J \leq J_{0}$ and $K \in \mathbb{Z}$, there is $v \in\left(2^{J} x, 2^{J} y\right)$, such that

$$
\psi^{\alpha}\left(2^{J} x-K\right)-\psi^{\alpha}\left(2^{J} y-K\right)=2^{J}(x-y) \frac{d \psi^{\alpha}}{d x}(v-K) .
$$

By using (2.20) and (2.21) we derive

$$
\begin{aligned}
\left|\frac{d \psi^{\alpha}}{d x}(v-K)\right| & \leq c_{2,5}(3+|v-K|)^{-2} \\
& \leq c_{2,5}\left(3+\left|2^{J} x-K\right|-\left|v-2^{J} x\right|\right)^{-2} \\
& \leq c_{2,5}\left(2+\left|2^{J} x-K\right|\right)^{-2}
\end{aligned}
$$

To estimate $S_{\alpha, 1}(x, y)$, we note that for all integers $J \leq-1$, (2.26) gives

$$
\left|\frac{d \psi^{\alpha}}{d x}(v-K)\right| \leq c_{2,5}\left(2+|K|-2^{J}|x|\right)^{-2} \leq c_{2,5}(1+|K|)^{-2} .
$$

So (2.22), (2.25), and (2.27) entail that

$$
S_{\alpha, 1}(x, y) \leq c_{2,6}|x-y|,
$$

where the constant $c_{2,6}=c_{2,5} \sum_{J=-\infty}^{-1} \sum_{K=-\infty}^{\infty} \frac{2^{J(1-\alpha)} \sqrt{\log (3+|J|+|K|)}}{(1+|K|)^{2}}$. 
Next we proceed to derive an upper bound for $S_{\alpha, 2}(x, y)$. Combining (2.23), (2.25), (2.26), and (2.15) yields

$$
\begin{aligned}
S_{\alpha, 2}(x, y) & \leq c_{2,5}|x-y| \sum_{J=0}^{J_{0}} \sum_{K=-\infty}^{\infty} \frac{2^{J(1-\alpha)} \sqrt{\log (3+J+|K|)}}{\left(2+\left|2^{J} x-K\right|\right)^{2}} \\
& =c_{2,5}|x-y| \sum_{J=0}^{J_{0}} \sum_{K=-\infty}^{\infty} \frac{2^{J(1-\alpha)} \sqrt{\log \left(3+J+\left|K+\left\lfloor 2^{J} x\right\rfloor\right|\right)}}{\left(2+\left|2^{J} x-\left\lfloor 2^{J} x\right\rfloor-K\right|\right)^{2}} \\
& \leq c_{2,5}|x-y| \sum_{J=0}^{J_{0}} \sum_{K=-\infty}^{\infty} \frac{2^{J(1-\alpha)} \sqrt{\log \left(3+J+2^{J}+|K|\right)}}{(1+|K|)^{2}} \\
& \leq c_{2,7}|x-y| \sum_{J=0}^{J_{0}} 2^{J(1-\alpha)} \sqrt{\log \left(3+J+2^{J}\right)} .
\end{aligned}
$$

In the above, $\lfloor z\rfloor$ denotes the integer part of $z$ and $0<c_{2,7}<\infty$ is a constant. Note that for any $J \in \mathbb{N}$,

$$
\sqrt{\log \left(3+J+2^{J}\right)} \leq \sqrt{\log \left(2^{J+2}+2^{J}\right)} \leq \sqrt{J+3} .
$$

It follows from (2.29), (2.30), and (2.21) that

$$
\begin{aligned}
S_{\alpha, 2}(x, y) & \leq c_{2,7}|x-y| \sum_{J=0}^{J_{0}} 2^{J(1-\alpha)} \sqrt{J+3} \\
& \leq c_{2,8}|x-y| 2^{\left(J_{0}+1\right)(1-\alpha)} \sqrt{J_{0}+3} \\
& \leq c_{2,9}|x-y|^{\alpha} \sqrt{3+\log \left(|x-y|^{-1}\right)} .
\end{aligned}
$$

Now, let us give an upper bound for $S_{\alpha, 3}(x, y)$. For every $z \in[0,1]$, let

$$
\theta_{J_{0}}(z)=\sum_{J=J_{0}+1}^{\infty} \sum_{K=-\infty}^{\infty} 2^{-J \alpha}\left|\psi^{\alpha}\left(2^{J} z-K\right)\right| \sqrt{\log (3+J+|K|)} .
$$

Then for every $x, y \in[0,1]$,

$$
S_{\alpha, 3}(x, y) \leq \theta_{J_{0}}(x)+\theta_{J_{0}}(y) .
$$

Thus, it is sufficient to bound $\theta_{J_{0}}(z)$, uniformly in $z \in[0,1]$. Note that for any fixed $J \in \mathbb{N}$ and any $z \in[0,1],(2.20)$ and (2.15) imply that

$$
\begin{aligned}
& \sum_{K=-\infty}^{\infty}\left|\psi^{\alpha}\left(2^{J} z-K\right)\right| \sqrt{\log (3+J+|K|)} \leq c_{2,5} \sum_{K=-\infty}^{\infty} \frac{\sqrt{\log (3+J+|K|)}}{\left(3+\left|2^{J} z-K\right|\right)^{2}} \\
& \quad=c_{2,5} \sum_{K=-\infty}^{\infty} \frac{\sqrt{\log \left(3+J+\mid K+\left\lfloor 2^{J} z||\right)\right.}}{\left(3+\left|2^{J} z-\left\lfloor 2^{J} z\right\rfloor-K\right|\right)^{2}} \\
& \quad \leq c_{2,5} \sum_{K=-\infty}^{\infty} \frac{\sqrt{\log \left(3+J+2^{J}+|K|\right)}}{(2+|K|)^{2}} \\
& \quad \leq c_{2,10} \sqrt{1+J},
\end{aligned}
$$


where $c_{2,10}$ is a finite constant. It follows from (2.34), (2.32) and some simple calculations that for every $z \in[0,1]$,

$$
\begin{aligned}
\theta_{J_{0}}(z) & \leq c_{2,10} \sum_{J=J_{0}+1}^{\infty} 2^{-J \alpha} \sqrt{1+J} \\
& \leq c_{2,10} \int_{J_{0}}^{\infty} 2^{-\alpha x} \sqrt{2+x} d x \\
& \leq c_{2,11} 2^{-J_{0} \alpha} \sqrt{1+J_{0}} .
\end{aligned}
$$

It is clear that (2.33), (2.35), and (2.21) entail that,

$$
S_{\alpha, 3}(x, y) \leq c_{2,12}|x-y|^{\alpha} \sqrt{\log \left(3+|x-y|^{-1}\right)},
$$

where $c_{2,12}>0$ is a constant independent of $x$ and $y$. Combining (2.28), (2.31), and (2.36) yields (2.18).

To prove (2.19), we observe that, by (2.10), $T_{\alpha}(x)=S_{\alpha}(x, 0)$ for every $x \in[0,1]$. Therefore, (2.19) follows from (2.18) immediately.

We are now in a position to prove Theorem 1.

Proof of Theorem 1. Observe that for any $s, t \in[0,1]^{N}$, one has that

$$
\begin{aligned}
& \left|B^{H}(t)-B^{H}(s)\right| \\
& \quad \leq \sum_{i=1}^{N}\left|B^{H}\left(s_{1}, \ldots, s_{i-1}, t_{i}, t_{i+1}, \ldots, t_{N}\right)-B^{H}\left(s_{1}, \ldots, s_{i-1}, s_{i}, t_{i+1}, \ldots, t_{N}\right)\right|
\end{aligned}
$$

with the convention that, when $i=1, B^{H}\left(s_{1}, \ldots, s_{i-1}, t_{i}, t_{i+1}, \ldots, t_{N}\right)=B^{H}(t)$ and when $i=N, B^{H}\left(s_{1}, \ldots, s_{i-1}, s_{i}, t_{i+1}, \ldots, t_{N}\right)=B^{H}(s)$. Another convention that will be used in the sequel is that, when $i=1, \prod_{l=1}^{i-1}\left|\widetilde{\psi}_{j_{l}, k_{l}}^{H_{l}}\left(s_{l}\right)\right|=1$ and when $i=N$, $\prod_{l=i+1}^{N}\left|\widetilde{\psi}_{j_{l}, k_{l}}^{H_{l}}\left(t_{l}\right)\right|=1$.

Let $\Omega_{1}^{*}$ be the event of probability 1 on which Proposition 3 and (2.14) hold. For every fixed integer $1 \leq i \leq N$, it follows from Propositions 2 and 3, Lemmas 1 and 2, (2.10) and (2.15) that for every $\omega \in \Omega_{1}^{*}$ and $s, t \in[0,1]^{N}$,

$$
\begin{aligned}
& \left|B^{H}\left(s_{1}, \ldots, s_{i-1}, t_{i}, t_{i+1}, \ldots, t_{N}\right)-B^{H}\left(s_{1}, \ldots, s_{i-1}, s_{i}, t_{i+1}, \ldots, t_{N}\right)\right| \\
& \quad \leq A_{4}\left(\prod_{l=1}^{i-1} T_{H_{l}}\left(s_{l}\right)\right) \times\left(\prod_{l=i+1}^{N} T_{H_{l}}\left(t_{l}\right)\right) \times S_{H_{i}}\left(s_{i}, t_{i}\right) \\
& \quad \leq A_{5}\left|s_{i}-t_{i}\right|^{H_{i}} \sqrt{\log \left(\left|s_{i}-t_{i}\right|^{-1}\right)},
\end{aligned}
$$

where the random variables $A_{4}>0$ and $A_{5}>0$ are of finite moments of any order. Finally, Theorem 1 follows from inequalities (2.37) and (2.38).

Now we prove Theorem 2.

Proof of Theorem 2. For any $\alpha \in(0,1)$, let $T_{\alpha}(x)$ be defined by (2.17). We first show that there is a constant $c_{2,13}>0$, depending on $\alpha$ only, such that for all $x \in \mathbb{R}$,

$$
T_{\alpha}(x) \leq c_{2,13}(1+|x|)^{\alpha} \sqrt{\log \log (3+|x|)} .
$$


It follows from (2.19) that the inequality (2.39) is satisfied when $|x| \leq 1$. It remains to show that it is also true when $|x|>1$. Our approach is similar to the proof of Lemma 2.

For any $|x|>1$, we choose an integer $J_{0} \geq 0$ such that

$$
2^{J_{0}} \leq|x|<2^{J_{0}+1}
$$

and then write $T_{\alpha}(x)$ as the sum of the following 3 parts:

$$
\begin{aligned}
& T_{\alpha, 1}(x)=\sum_{J=-\infty}^{-J_{0}-1} \sum_{K=-\infty}^{\infty} 2^{-J \alpha}\left|\widetilde{\psi}_{J, K}^{\alpha}(x)\right| \sqrt{\log (3+|J|+|K|)}, \\
& T_{\alpha, 2}(x)=\sum_{J=-J_{0}}^{-1} \sum_{K=-\infty}^{\infty} 2^{-J \alpha}\left|\widetilde{\psi}_{J, K}^{\alpha}(x)\right| \sqrt{\log (3+|J|+|K|)}
\end{aligned}
$$

and

$$
T_{\alpha, 3}(x)=\sum_{J=0}^{\infty} \sum_{K=-\infty}^{\infty} 2^{-J \alpha}\left|\widetilde{\psi}_{J, K}^{\alpha}(x)\right| \sqrt{\log (3+|J|+|K|)} .
$$

First, let us derive an upper bound for $T_{\alpha, 1}(x)$. For any integers $-\infty<J \leq-J_{0}-1$ and $K \in \mathbb{Z}$, (2.10), the Mean-Value Theorem and (2.20) imply that

$$
\left|\widetilde{\psi}_{J, K}^{\alpha}(x)\right| \leq \frac{c_{2,5} 2^{J}|x|}{(3+|v-K|)^{2}} \leq \frac{c_{2,5} 2^{J}|x|}{(2+|K|)^{2}} .
$$

Putting (2.44) into (2.41) yields

$$
T_{\alpha, 1}(x) \leq c_{2,14}|x|
$$

for some finite constant $c_{2,14}>0$.

To derive an upper bound for $T_{\alpha, 2}(x)$, we note that

$$
T_{\alpha, 2}(x) \leq R_{\alpha, 2}(x)+R_{\alpha, 2}(0),
$$

where

$$
R_{\alpha, 2}(x)=\sum_{J=1}^{J_{0}} \sum_{K=-\infty}^{\infty} 2^{J \alpha}\left|\psi^{\alpha}\left(2^{-J} x-K\right)\right| \sqrt{\log (3+J+|K|)} .
$$

Applying (2.20) with $n=0$ and (2.15), we have

$$
\begin{aligned}
R_{2, \alpha}(x) & \leq c_{2,5} \sum_{J=1}^{J_{0}} \sum_{K=-\infty}^{\infty} \frac{2^{J \alpha} \sqrt{\log (3+J+|K|)}}{\left(3+\left|2^{-J} x-K\right|\right)^{2}} \\
& \leq c_{2,15} \sum_{J=1}^{J_{0}} 2^{J \alpha} \sqrt{\log \left(4+J+2^{-J}|x|\right)} .
\end{aligned}
$$

Hence, with some elementary computation, we have

$$
\begin{aligned}
R_{\alpha, 2}(x) & \leq c_{2,16} \int_{0}^{J_{0}} 2^{\alpha t} \sqrt{\log \left(5+t+2^{-t}|x|\right)} d x \\
& \leq c_{2,17} 2^{J_{0} \alpha} \sqrt{\log \left(5+J_{0}+2^{-J_{0}}|x|\right)} \\
& \leq c_{2,18}(1+|x|)^{\alpha} \sqrt{\log \log (3+|x|)},
\end{aligned}
$$


where the last inequality follows from (2.40).

Similarly, (2.20) and (2.15) imply that

$$
\begin{aligned}
R_{\alpha, 2}(0) & =\sum_{J=1}^{J_{0}} \sum_{K=-\infty}^{\infty} 2^{J \alpha}\left|\psi^{\alpha}(-K)\right| \sqrt{\log (3+J+|K|)} \\
& \leq c_{2,19} \sum_{J=1}^{J_{0}} 2^{J \alpha} \sqrt{\log (3+J)} \\
& \leq c_{2,20}(1+|x|)^{\alpha} \sqrt{\log (\log (3+|x|))}
\end{aligned}
$$

Combining (2.46), (2.47), and (2.50) we obtain that for any $|x|>1$,

$$
T_{\alpha, 2}(x) \leq c_{2,21}(1+|x|)^{\alpha} \sqrt{\log \log (3+|x|)} .
$$

Now, let us derive an upper bound for $T_{\alpha, 3}(x)$. It follows from (2.43) and (2.10) that for all $x \in \mathbb{R}$,

$$
T_{\alpha, 3}(x) \leq R_{\alpha, 3}(x)+R_{\alpha, 3}(0)
$$

where for any $x \in \mathbb{R}$,

$$
R_{\alpha, 3}(x)=\sum_{J=0}^{\infty} \sum_{K=-\infty}^{\infty} 2^{-J \alpha}\left|\psi^{\alpha}\left(2^{J} x-K\right)\right| \sqrt{\log (3+J+|K|)} .
$$

Using (2.20) again one obtains that for all $x \in \mathbb{R}$,

$$
\begin{aligned}
R_{\alpha, 3}(x) & \leq c_{2,5} \sum_{J=0}^{\infty} \sum_{K=-\infty}^{\infty} \frac{2^{-J \alpha} \sqrt{\log (3+J+|K|)}}{\left(3+\left|2^{J} x-K\right|\right)^{2}} \\
& =c_{2,5} \sum_{J=0}^{\infty} \sum_{K=-\infty}^{\infty} \frac{2^{-J \alpha} \sqrt{\log \left(3+J+\left|\left\lfloor 2^{J} x\right\rfloor\right|+|K|\right)}}{\left(3+\left|2^{J} x-\left\lfloor 2^{J} x\right\rfloor-K\right|\right)^{2}} \\
& \leq c_{2,22} \sum_{J=0}^{\infty} 2^{-J \alpha} \sqrt{\log \left(3+J+\left|\left\lfloor 2^{J} x\right\rfloor\right|\right)} .
\end{aligned}
$$

Note that for any $x \in \mathbb{R}$,

$$
\sqrt{\log \left(3+J+\left|\left\lfloor 2^{J} x\right\rfloor\right|\right)} \leq \sqrt{\log \left(3+J+2^{J}\right)}+\sqrt{\log (3+|x|)} .
$$

It follows from (2.52), (2.53), (2.54), and (2.55) that for any $x \in \mathbb{R}$,

$$
T_{\alpha, 3}(x) \leq c_{2,23} \sqrt{\log (3+|x|)} .
$$

It is clear that (2.56), (2.51), and (2.45) entail (2.39). Finally, (2.11), (2.9), (2.39), (2.15), and Lemma 1 imply that there is a random variable $A_{6}>0$ of finite moments of any order such that for all $\omega \in \Omega_{1}^{*}$ and $t=\left(t_{1}, \ldots, t_{N}\right) \in \mathbb{R}^{N}$,

$$
\left|B^{H}(t, \omega)\right| \leq A_{6} \prod_{j=1}^{N} T_{H_{j}}\left(t_{j}\right) \leq c_{2,24} A_{6} \prod_{j=1}^{N}\left(1+\left|t_{j}\right|\right)^{H_{j}} \sqrt{\log \log \left(3+\left|t_{j}\right|\right)} .
$$


This finishes the proof of Theorem 2 with $A_{2}=c_{2,24} A_{6}$.

Remark 2. Observe that while proving Theorem 2, we have obtained the following result which will be used in the proof of Proposition 4. Namely, there is a random variable $A_{2}>0$ of finite moments of any order such that for all $n \geq 1, t=\left(t_{1}, \ldots, t_{N}\right) \in \mathbb{R}^{N}$ and $\omega \in \Omega_{1}^{*}$, one has

$$
\begin{aligned}
\left|B_{n}^{H}(t, \omega)\right| & \leq \sum_{(j, k) \in \mathbb{Z}^{N} \times \mathbb{Z}^{N}} 2^{-\langle j, H\rangle}\left|\epsilon_{j, k}(\omega)\right|\left|\widetilde{\Psi}_{j, k}(t)\right| \\
& \leq A_{2}(\omega) \prod_{j=1}^{N}\left(1+\left|t_{j}\right|\right)^{H_{j}} \sqrt{\log \log \left(3+\left|t_{j}\right|\right)} .
\end{aligned}
$$

The rest of this section is devoted to the proof of Theorem 3. First, we need to fix some more notation.

- For any $\lambda=\left(\lambda_{1}, \lambda_{2}, \ldots, \lambda_{N}\right) \in \mathbb{R}^{N}$ and for each integer $1 \leq n \leq N$, we denote by $\widehat{\lambda}_{n}$ the vector of $\mathbb{R}^{N-1}$ defined as

$$
\widehat{\lambda}_{n}=\left(\lambda_{1}, \ldots, \lambda_{n-1}, \lambda_{n+1}, \ldots, \lambda_{N}\right)
$$

with the convention that $\widehat{\lambda}_{1}=\left(\lambda_{2}, \lambda_{3}, \ldots, \lambda_{N}\right)$ and $\widehat{\lambda}_{N}=\left(\lambda_{1}, \lambda_{2}, \ldots, \lambda_{N-1}\right)$. For convenience, we may sometimes write $\lambda$ as $\left(\lambda_{n}, \widehat{\lambda}_{n}\right)$ [see, e.g., (2.69)].

- For any $H=\left(H_{1}, \ldots, H_{N}\right) \in(0,1)^{N}, j=\left(j_{1}, \ldots, j_{N}\right) \in \mathbb{Z}^{N}, k=$ $\left(k_{1}, \ldots, k_{N}\right) \in \mathbb{Z}^{N}$ and $t=\left(t_{1}, \ldots, t_{N}\right) \in \mathbb{R}^{N}$, we set

$$
\widetilde{\Psi}_{\widehat{j}_{n}, \widehat{k}_{n}}^{\left(\widehat{H}_{n}\right)}\left(\widehat{t}_{n}\right)=\left(\prod_{l=1}^{n-1} \widetilde{\psi}_{j_{l}, k_{l}}^{H_{l}}\left(t_{l}\right)\right)\left(\prod_{l=n+1}^{N} \widetilde{\psi}_{j_{l}, k_{l}}^{H_{l}}\left(t_{l}\right)\right)
$$

with the convention that $\widetilde{\Psi}_{\widehat{j}_{1}, \widehat{k}_{1}}^{\left(\widehat{H}_{1}\right)}\left(\widehat{t}_{1}\right)=\prod_{l=2}^{N} \widetilde{\psi}_{j_{l}, k_{l}}^{H_{l}}\left(t_{l}\right)$ and $\widetilde{\Psi}_{\widehat{j}_{N}, \widehat{k}_{N}}^{\left(\widehat{H}_{N}\right)}\left(\widehat{t}_{N}\right)=$ $\prod_{l=1}^{N-1} \widetilde{\psi}_{j_{l}, k_{l}}^{H_{l}}\left(t_{l}\right)$. Recall that the function $\widetilde{\psi}_{j_{l}, k_{l}}^{H_{l}}\left(t_{l}\right)$ is defined in (2.10).

Let us now introduce a wavelet transform that allows us to construct a sequence of independent and identically distributed fractional Brownian sheets on $\mathbb{R}^{N-1}$ starting from a fractional Brownian sheet on $\mathbb{R}^{N}$.

Proposition 4. Let $B^{H}=\left\{B^{H}(t), t \in \mathbb{R}^{N}\right\}$ be an $(N, 1)$-fractional Brownian sheet with index $H=\left(H_{1}, \ldots, H_{N}\right)$. For every $n \in\{1, \ldots, N\},\left(j_{n}, k_{n}\right) \in \mathbb{Z} \times \mathbb{Z}$ and

$$
\widehat{t}_{n}=\left(t_{1}, \ldots, t_{n-1}, t_{n+1}, \ldots, t_{N}\right) \in \mathbb{R}^{N-1},
$$

we define

$$
C_{j_{n}, k_{n}}\left(\widehat{t}_{n}\right)=2^{j_{n}\left(1+H_{n}\right)} \int_{\mathbb{R}} B^{H}(t) \psi^{-H_{n}}\left(2^{j_{n}} t_{n}-k_{n}\right) d t_{n},
$$

where $\psi^{-H_{n}}$ is the wavelet introduced in (2.7). Then $\left(C_{j_{n}, k_{n}}\right)_{\left(j_{n}, k_{n}\right) \in \mathbb{Z} \times \mathbb{Z}}$ is a sequence of independent and identically distributed fractional Brownian sheets on $\mathbb{R}^{N-1}$ with index $\widehat{H}_{n}$.

Proof. Note that Property (b) of Lemarié-Meyer wavelets and (2.8) imply that $\widehat{\psi}^{-} H_{n}(0)=$ 0 . Therefore one has that,

$$
\int_{\mathbb{R}} \psi^{-H_{n}}\left(2^{j_{n}} t_{n}-k_{n}\right) d t_{n}=0
$$


For every $(p, q) \in \mathbb{Z}^{N} \times \mathbb{Z}^{N}$ and for every $\widehat{t}_{n} \in \mathbb{R}^{N-1}$, define

$$
I\left(\widehat{t}_{n} ; p, q ; j_{n}, k_{n}\right)=2^{j_{n}} \int_{\mathbb{R}} \widetilde{\Psi}_{p, q}^{(H)}(t) \psi^{-H_{n}}\left(2^{j_{n}} t_{n}-k_{n}\right) d t_{n} .
$$

We claim that

$$
I\left(\widehat{t}_{n} ; p, q ; j_{n}, k_{n}\right)=\delta\left(p_{n}, q_{n} ; j_{n}, k_{n}\right) \widetilde{\Psi}_{\widehat{p}_{n}, \widehat{q}_{n}}^{\left(\widehat{t}_{n}\right.}\left(\widehat{t}_{n}\right),
$$

where $\delta\left(p_{n}, q_{n} ; j_{n}, k_{n}\right)=1$, if $\left(p_{n}, q_{n}\right)=\left(j_{n}, k_{n}\right)$ and $\delta\left(p_{n}, q_{n} ; j_{n}, k_{n}\right)=0$ otherwise. To verify (2.63), note that (2.9), (2.10), (2.59), and (2.61) imply that

$$
\begin{aligned}
2^{j_{n}} & \int_{\mathbb{R}} \widetilde{\Psi}_{p, q}^{(H)}(t) \psi^{-H_{n}}\left(2^{j_{n}} t_{n}-k_{n}\right) d t_{n} \\
\quad= & 2^{j_{n}} \widetilde{\Psi}_{\widehat{p}_{n}, \widehat{\mathcal{G}}_{n}}^{\left(\widehat{q}_{n}\right.}\left(\widehat{t}_{n}\right) \int_{\mathbb{R}} \psi^{H_{n}}\left(2^{p_{n}} t_{n}-q_{n}\right) \psi^{-H_{n}}\left(2^{j_{n}} t_{n}-k_{n}\right) d t_{n} .
\end{aligned}
$$

Since the functions $\psi^{H_{n}}$ and $\psi^{-H_{n}}$ are real-valued, it follows from Parseval's formula, (2.8) and the orthonormality of the Lemarié-Meyer wavelets $2^{J / 2} \psi\left(2^{J} x-K\right), J \in \mathbb{Z}, K \in \mathbb{Z}$, that

$$
\begin{aligned}
2^{j_{n}} & \int_{\mathbb{R}} \psi^{H_{n}}\left(2^{p_{n}} t_{n}-q_{n}\right) \psi^{-H_{n}}\left(2^{j_{n}} t_{n}-k_{n}\right) d t_{n} \\
& =2^{-p_{n}} \int_{\mathbb{R}} e^{-i\left(2^{-p_{n}} q_{n}-2^{-j_{n}} k_{n}\right) \xi_{n}} \widehat{\psi^{H_{n}}}\left(2^{-p_{n}} \xi_{n}\right) \overline{\widehat{\psi^{-H_{n}}}\left(2^{-j_{n}} \xi_{n}\right)} d \xi_{n} \\
& =2^{\left(H_{n}+1 / 2\right)\left(p_{n}-j_{n}\right)-p_{n}} \int_{\mathbb{R}} e^{-i\left(2^{-p_{n}} q_{n}-2^{-j_{n}} k_{n}\right) \xi_{n}} \widehat{\psi}\left(2^{-p_{n}} \xi_{n}\right) \widehat{\widehat{\psi}\left(2^{-j_{n}} \xi_{n}\right)} d \xi_{n} \\
& =2^{\left(H_{n}+1 / 2\right)\left(p_{n}-j_{n}\right)+j_{n}} \int_{\mathbb{R}} \psi\left(2^{p_{n}} t_{n}-q_{n}\right) \psi\left(2^{j_{n}} t_{n}-k_{n}\right) d t_{n} \\
& =2^{\left(H_{n}+1 / 2\right)\left(p_{n}-j_{n}\right)} \delta\left(p_{n}, q_{n} ; j_{n}, k_{n}\right) .
\end{aligned}
$$

Combining (2.64) and (2.65) yields (2.63).

Next it follows from Proposition 3, (2.60), (2.62), and (2.63), that almost surely, for every $\widehat{t}_{n} \in \mathbb{R}^{N-1}$,

$$
\begin{aligned}
C_{j_{n}, k_{n}}\left(\widehat{t}_{n}\right) & =\sum_{(p, q) \in \mathbb{Z}^{N} \times \mathbb{Z}^{N}} 2^{-\langle p, H\rangle} \epsilon_{p, q} I\left(\widehat{t}_{n} ; p, q ; j_{n}, k_{n}\right) \\
& =\sum_{\left(\widehat{p}_{n}, \widehat{q}_{n}\right) \in \mathbb{Z}^{N-1} \times \mathbb{Z}^{N-1}} 2^{-\left\langle\widehat{p}_{n}, \widehat{H}_{n}\right\rangle} \epsilon_{\left(j_{n}, \widehat{p}_{n}\right),\left(k_{n}, \widehat{q}_{n}\right)} \widetilde{\Psi}_{\widehat{p}_{n}, \widehat{q}_{n}}^{\left(\widehat{q}_{n}\right)}\left(\widehat{t_{n}}\right) .
\end{aligned}
$$

Observe that we are allowed to interchange the order of integration and summation in deriving the first equality in (2.66) because the function $t_{n} \mapsto \psi^{-H_{n}}\left(2^{j_{n}} t_{n}-k_{n}\right)$ belongs to $\mathcal{S}(\mathbb{R})$, the partial-sum processes $B_{m}^{H}(t)$ [which are defined in (2.12)] converge to $B^{H}(t)$ uniformly on all compact sets and because of (2.57). Also, observe that Propositions 2 and 3 entail that, with probability 1 , the series (2.66) is uniformly convergent in $\widehat{t}_{n}$, on any compact of $\mathbb{R}^{N-1}$ and that the Gaussian field $\left\{C_{j_{n}, k_{n}}\left(\widehat{t_{n}}\right), \widehat{t_{n}} \in \mathbb{R}^{N-1}\right\}$ is a fractional Brownian sheet on $\mathbb{R}^{N-1}$, with index $\widehat{H}_{n}$. Finally, observe that the Gaussian random


and have the same distribution, so are the fractional Brownian sheets $\left\{C_{j_{n}, k_{n}}\left(\widehat{t_{n}}\right), \widehat{t}_{n} \in\right.$ $\left.\mathbb{R}^{N-1}\right\},\left(\left(j_{n}, k_{n}\right) \in \mathbb{Z} \times \mathbb{Z}\right)$. 
Let us show that the increments of the Gaussian field $\left\{C_{j_{n}, k_{n}}\left(\widehat{t_{n}}\right), \widehat{t_{n}} \in[0,1]^{N-1}\right\}$ can be controlled uniformly in the indices $j_{n}$ and $k_{n}$.

Lemma 3. Let $\Omega_{1}^{*}$ be the event of probability 1 in Theorem 1 . For every $1 \leq n \leq N$ and $x, y \in[0,1]^{N}$, let

$$
\tau_{n}\left(\widehat{x}_{n}, \widehat{y}_{n}\right)=\sum_{l \neq n}\left|x_{l}-y_{l}\right|^{H_{l}} \sqrt{\log \left(3+\left|x_{l}-y_{l}\right|^{-1}\right)} .
$$

Then there is a random variable $A_{7}>0$ of finite moments of any order, such that for every $j_{n} \in \mathbb{N}, k_{n} \in\left\{0,1, \ldots, 2^{j_{n}}\right\}, \widehat{x}_{n} \in[0,1]^{N-1}, \widehat{y}_{n} \in[0,1]^{N-1}$ and $\omega \in \Omega_{1}^{*}$,

$$
\left|C_{j_{n}, k_{n}}\left(\widehat{x}_{n}, \omega\right)-C_{j_{n}, k_{n}}\left(\widehat{y}_{n}, \omega\right)\right| \leq A_{7}(\omega) 2^{j_{n} H_{n}} \tau_{n}\left(\widehat{x}_{n}, \widehat{y}_{n}\right)
$$

Proof. Following the same lines as in the proofs of Theorems 1 and 2, one can show that there is a random variable $A_{8}>0$, of finite moments of any order, such that for every $t_{n} \in \mathbb{R}, \widehat{x}_{n} \in[0,1]^{N-1}, \widehat{y}_{n} \in[0,1]^{N-1}$ and $\omega \in \Omega_{1}^{*}$, one has

$$
\left|B^{H}\left(t_{n}, \widehat{x}_{n}, \omega\right)-B^{H}\left(t_{n}, \widehat{y}_{n}, \omega\right)\right| \leq A_{8}(\omega) \tau_{n}\left(\widehat{x}_{n}, \widehat{y}_{n}\right)\left(1+\left|t_{n}\right|\right)^{H_{n}} \sqrt{\log \log \left(3+\left|t_{n}\right|\right)}
$$

It follows from (2.60) and (2.69) that for all $j_{n} \in \mathbb{N}, k_{n} \in\left\{0,1, \ldots, 2^{j_{n}}\right\}, \widehat{x}_{n} \in$ $[0,1]^{N-1}$ and $\widehat{y}_{n} \in[0,1]^{N-1}$,

$$
\begin{aligned}
& \left|C_{j_{n}, k_{n}}\left(\widehat{x}_{n}, \omega\right)-C_{j_{n}, k_{n}}\left(\widehat{y}_{n}, \omega\right)\right| \\
& \quad \leq 2^{j_{n}\left(1+H_{n}\right)} \int_{\mathbb{R}}\left|B^{H}\left(t_{n}, \widehat{x}_{n}, \omega\right)-B^{H}\left(t_{n}, \widehat{y}_{n}, \omega\right)\right|\left|\psi^{-H_{n}}\left(2^{j_{n}} t_{n}-k_{n}\right)\right| d t_{n} \\
& \quad \leq A_{8}(\omega) 2^{j_{n}\left(1+H_{n}\right)} \tau_{n}\left(\widehat{x}_{n}, \widehat{y}_{n}\right) \int_{\mathbb{R}}\left(1+\left|t_{n}\right|\right)^{H_{n}} \sqrt{\log \log \left(3+\left|t_{n}\right|\right)}\left|\psi^{-H_{n}}\left(2^{j_{n}} t_{n}-k_{n}\right)\right| d t_{n},
\end{aligned}
$$

where the last integral is finite since $\psi^{-H_{n}}$ belongs to $\mathcal{S}(\mathbb{R})$. Moreover, the integral

$$
I\left(j_{n}, k_{n}\right)=2^{j_{n}} \int_{\mathbb{R}}\left(1+\left|t_{n}\right|\right)^{H_{n}} \sqrt{\log \log \left(3+\left|t_{n}\right|\right)}\left|\psi^{-H_{n}}\left(2^{j_{n}} t_{n}-k_{n}\right)\right| d t_{n}
$$

can be bounded independently of $j_{n} \in \mathbb{N}$ and $k_{n} \in\left\{0,1, \ldots, 2^{j_{n}}\right\}$. Indeed, by setting $u=2^{j_{n}} t_{n}-k_{n}$, we derive that

$$
I\left(j_{n}, k_{n}\right) \leq \int_{\mathbb{R}}(2+|u|)^{H_{n}} \sqrt{\log \log (4+|u|)}\left|\psi^{-H_{n}}(u)\right| d u<\infty .
$$

Thus, (2.69) follows from (2.70) and (2.71).

In the following, without loss of generality, we will suppose that

$$
H_{1}=\min \left\{H_{1}, \ldots, H_{N}\right\} \text {. }
$$

Lemma 4. Let $\Omega_{1}^{*}$ be the event with probability 1 in Theorem 1 . If there exist some $\epsilon>0$, $s=\left(s_{1}, \widehat{s}_{1}\right) \in(0,1] \times(0,1]^{N-1}$ and $\omega \in \Omega_{1}^{*}$ such that

$$
\limsup _{|h| \rightarrow 0} \frac{\left|B^{H}(s+h, \omega)-B^{H}(s, \omega)\right|}{\sigma(s+h, s)^{1+\epsilon}}<\infty,
$$


then there is a constant $c_{2,25}>0$ (only depending on $s, \epsilon$ and $\omega$ ) such that for every $j_{1} \in \mathbb{N}$ and $k_{1} \in\left\{0,1, \ldots, 2^{j_{1}}\right\}$,

$$
\left|C_{j_{1}, k_{1}}\left(\widehat{s}_{1}, \omega\right)\right| \leq c_{2,25} 2^{-j_{1} \epsilon H_{1}}\left(1+\left|2^{j_{1}} s_{1}-k_{1}\right|\right)^{(1+\epsilon) H_{1}} .
$$

Proof. When (2.73) holds, we can find two constants $c_{2,26}>0$ and $\eta>0$ [depending on $s, \epsilon$ and $\omega]$ such that for all $h \in \mathbb{R}^{N}$ satisfying $|h| \leq \eta$,

$$
\left|B^{H}(s+h, \omega)-B^{H}(s, \omega)\right| \leq c_{2,26} \sigma(s+h, s)^{1+\epsilon} .
$$

In particular, for $h=\left(h_{1}, 0, \ldots, 0\right)$ with $\left|h_{1}\right| \leq \eta$, we have

$$
\left|B^{H}\left(s_{1}+h_{1}, \widehat{s}_{1}, \omega\right)-B^{H}\left(s_{1}, \widehat{s}_{1}, \omega\right)\right| \leq c_{2,26}\left|h_{1}\right|^{H_{1}(1+\epsilon)} .
$$

Observe that Theorem 2 implies that by increasing the value of $c_{2,26},(2.76)$ holds for all $h_{1} \in \mathbb{R}$. Hence, it follows from (2.60), (2.61), and (2.76) that for every $j_{n} \in \mathbb{N}$ and $k_{n} \in\left\{0,1, \ldots, 2^{j_{n}}\right\}$,

$$
\begin{aligned}
\left|C_{j_{1}, k_{1}}\left(\widehat{s}_{1}, \omega\right)\right| & \leq 2^{j_{1}\left(1+H_{1}\right)} \int_{\mathbb{R}}\left|B^{H}\left(t_{1}, \widehat{s}_{1}\right)-B^{H}\left(s_{1}, \widehat{s}_{1}\right)\right|\left|\psi^{-H_{1}}\left(2^{j_{1}} t_{1}-k_{1}\right)\right| d t_{1} \\
& \leq c_{2,26} 2^{j_{1}\left(1+H_{1}\right)} \int_{\mathbb{R}}\left|t_{1}-s_{1}\right|^{H_{1}(1+\epsilon)}\left|\psi^{-H_{1}}\left(2^{j_{1}} t_{1}-k_{1}\right)\right| d t_{1} .
\end{aligned}
$$

Setting $u=2^{j_{1}} t_{1}-k_{1}$ in this last integral, one obtains that

$$
\begin{aligned}
\left|C_{j_{1}, k_{1}}\left(\widehat{s}_{1}, \omega\right)\right| \leq & c_{2,26} 2^{j_{1} H_{1}} \int_{\mathbb{R}}\left|2^{-j_{1}} u+2^{-j_{1}} k_{1}-s_{1}\right|^{H_{1}(1+\epsilon)}\left|\psi^{-H_{1}}(u)\right| d u \\
\leq & c_{2,27} 2^{-j_{1} \epsilon H_{1}} \int_{\mathbb{R}}|u|^{H_{1}(1+\epsilon)}\left|\psi^{-H_{1}}(u)\right| d u \\
& +c_{2,27} 2^{-j_{1} \epsilon H_{1}}\left|2^{j_{1}} s_{1}-k_{1}\right|^{H_{1}(1+\epsilon)} \int_{\mathbb{R}}\left|\psi^{-H_{1}}(u)\right| d u \\
\leq & c_{2,25} 2^{-j_{1} \epsilon H_{1}}\left(1+\left|2^{j_{1}} s_{1}-k_{1}\right|\right)^{(1+\epsilon) H_{1}},
\end{aligned}
$$

for some finite constant $c_{2,25}>0$. This proves (2.74).

The proof of Theorem 3 mainly relies on the following technical lemma, which is, to a certain extent, inspired by Lemma 4.1 in Ayache, Jaffard, and Taqqu [5].

Lemma 5. There is an event $\Omega_{3}^{*}$ with probability 1 satisfying the following property: For all arbitrarily small $\eta>0, \epsilon>0$ and for all $\omega \in \Omega_{3}^{*}$, there exist a real number $v>0$ and an integer $j_{1,0} \geq 0$ such that for all integers $j_{1} \geq j_{1,0}, \widehat{k}_{1} \in\left\{0,1, \ldots, 3^{j_{1}}\right\}^{N-1}$ with $3^{-j_{1}} \widehat{k}_{1} \in[\eta, 1]^{N-1}$ and for all $t_{1} \in[0,1]$, there exists $k_{1}\left(j_{1}\right) \in\left\{0,1, \ldots, 2^{j_{1}}\right\}$ such that

$$
\left|t_{1}-2^{-j_{1}} k_{1}\left(j_{1}\right)\right| \leq 2^{-j_{1}\left(1-H_{1} \epsilon\right)+1}
$$

and

$$
\left|C_{j_{1}, k_{1}\left(j_{1}\right)}\left(3^{-j_{1}} \widehat{k}_{1}, \omega\right)\right| \geq v .
$$

Proof. Let us fix two constants $\epsilon, \eta \in(0,1)$. Since the distribution of the fractional Brownian sheet $\left\{C_{j_{1}, k_{1}}\left(\widehat{x}_{1}\right), \widehat{x}_{1} \in[\eta, 1]^{N-1}\right\}$ is independent of the indices $j_{1}$ and $k_{1}$ (see 
Proposition 4), there is a constant $v_{\eta}>0$, depending on $\eta$ only, such that for all $\widehat{x}_{1} \in$ $[\eta, 1]^{N-1}, j_{1} \in \mathbb{N}$ and $k_{1} \in\left\{0,1, \ldots, 2^{j_{1}}\right\}$,

$$
\sqrt{\operatorname{Var}\left(C_{j_{1}, k_{1}}\left(\widehat{x}_{1}\right)\right)} \geq v_{\eta}
$$

Thus, for all $j_{1} \in \mathbb{N}, k_{1} \in\left\{0,1, \ldots, 2^{j_{1}}\right\}$ and $\widehat{x}_{1} \in[\eta, 1]^{N-1}$,

$$
\mathbb{P}\left(\left|C_{j_{1}, k_{1}}\left(\widehat{x}_{1}\right)\right| \leq v_{\eta}\right) \leq \sqrt{\frac{2}{\pi}} .
$$

For any $j_{1} \in \mathbb{N}$, we set

$$
D_{\eta, j_{1}}=\left\{2^{-j_{1}} \widehat{k}_{1}: \widehat{k}_{1} \in\left\{0,1, \ldots, 3^{j_{1}}\right\}^{N-1} \text { and } 3^{-j_{1}} \widehat{k}_{1} \in[\eta, 1]^{N-1}\right\}
$$

and

$$
L_{\epsilon, j_{1}}=\left\lfloor\frac{2^{j_{1}}}{\left\lfloor 2^{j_{1} H_{1} \epsilon}\right\rfloor}\right\rfloor,
$$

where $\lfloor x\rfloor$ is the integer part of $x$. Observe that each $k_{1} \in\left\{0,1, \ldots, 2^{j_{1}}\right\}$ can be written as

$$
k_{1}=\left\lfloor 2^{j_{1} H_{1} \epsilon}\right\rfloor q+r,
$$

where $q \in\left\{0,1, \ldots, L_{\epsilon, j_{1}}\right\}$ and $r \in\left\{0,1, \ldots,\left\lfloor 2^{j_{1} H_{1} \epsilon}\right\rfloor-1\right\}$. Also observe that for all $t_{1} \in[0,1]$ and $j_{1} \in \mathbb{N}$, there is $\bar{q}_{j_{1}} \in\left\{0,1, \ldots, L_{\epsilon, j_{1}}\right\}$ and $\bar{r}_{j_{1}} \in\left\{0,1, \ldots,\left\lfloor 2^{j_{1} H_{1} \epsilon}\right\rfloor-1\right\}$ such that

$$
\left|t_{1}-\frac{\left\lfloor 2^{j_{1} H_{1} \epsilon}\right\rfloor \bar{q}_{j_{1}}+\bar{r}_{j_{1}}}{2^{j_{1}}}\right| \leq 2^{-j_{1}}
$$

Hence, we have for all $r_{j_{1}} \in\left\{0,1, \ldots,\left\lfloor 2^{j_{1} H_{1} \epsilon}\right\rfloor-1\right\}$,

$$
\left|t_{1}-\frac{\left\lfloor 2^{j_{1} H_{1} \epsilon}\right\rfloor \bar{q}_{j_{1}}+r_{j_{1}}}{2^{j_{1}}}\right| \leq 2^{-j_{1}\left(1-\epsilon H_{1}\right)+1}
$$

and (2.77) follows from this last inequality.

Now we consider the event $E_{\eta, \epsilon, j_{1}}$ defined by

$$
E_{\eta, \epsilon, j_{1}}=\bigcup_{\widehat{d}_{1} \in D_{\eta, j_{1}}} \bigcup_{q=0}^{L_{\epsilon, j_{1}}} \bigcap_{r=0}^{\left\lfloor 2^{j_{1} H_{1} \epsilon}\right\rfloor-1}\left\{\left|C_{j_{1},\left\lfloor 2^{j_{1} H_{1} \epsilon}\right\rfloor q+r}\left(\widehat{d}_{1}\right)\right| \leq v_{\eta}\right\} .
$$

It follows from the independence of the random variables $C_{j_{1},\left\lfloor 2^{j_{1} H_{1} \epsilon}\right\rfloor q+r}\left(\widehat{d}_{1}\right)$, (see Proposition 4$),(2.80),(2.81)$, and (2.82) that

$$
\begin{aligned}
\mathbb{P}\left(E_{\eta, \epsilon, j_{1}}\right) & \leq\left(3^{j_{1}}+1\right)^{N-1}\left(L_{\epsilon, j_{1}}+1\right)\left(\frac{2}{\pi}\right)^{2^{j_{1} H_{1} \epsilon}-1} \\
& \leq \exp \left(c_{2,28} j_{1}+\left(\log \frac{2}{\pi}\right)\left(2^{j_{1} H_{1} \epsilon}-1\right)\right) .
\end{aligned}
$$

Since $\sum_{j_{1}=0}^{\infty} \mathbb{P}\left(E_{\eta, \epsilon, j_{1}}\right)<\infty$, the Borel-Cantelli Lemma implies that

$$
\mathbb{P}\left(\bigcup_{J_{1}=0}^{\infty} \bigcap_{j_{1}=J_{1}}^{\infty} E_{\eta, \epsilon, j_{1}}^{c}\right)=1
$$


where $E_{\eta, \epsilon, j_{1}}^{c}$ denotes the complementary of the event $E_{\eta, \epsilon, j_{1}}$.

Finally, we take

$$
\Omega_{3}^{*}=\bigcap_{m \in \mathbb{N}} \bigcap_{n \in \mathbb{N}} \bigcup_{J_{1}=0}^{\infty} \bigcap_{j_{1}=J_{1}}^{\infty} E_{\frac{1}{m+1}, \frac{1}{n+1}, j_{1}}^{c}
$$

and it has the desired properties of Lemma 5 .

The following lemma is a consequence of Lemmas 3 and 5.

Lemma 6. Let $\Omega_{2}^{*}=\Omega_{1}^{*} \cap \Omega_{3}^{*}$. Then, for all $\epsilon>0$ small enough, $\left(t_{1}, \widehat{t}_{1}\right) \in(0,1] \times$ $(0,1]^{N-1}$ and all $\omega \in \Omega_{2}^{*}$, there exist $v>0$ and an integer $j_{1,1} \geq 0$ satisfying the following property: For every integer $j_{1} \geq j_{1,1}$, there exists $k_{1}\left(j_{1}\right) \in\left\{0,1, \ldots, 2^{j_{1}}\right\}$ such that

$$
\left|t_{1}-2^{-j_{1}} k_{1}\left(j_{1}\right)\right| \leq 2^{-j_{1}\left(1-H_{1} \epsilon\right)+1}
$$

and

$$
\left|C_{j_{1}, k_{1}\left(j_{1}\right)}\left(\widehat{t}_{1}, \omega\right)\right| \geq v / 2 .
$$

Proof. Let us fix $t=\left(t_{1}, \widehat{t}_{1}\right) \in(0,1] \times(0,1]^{N-1}, \epsilon>0$ small enough and $\omega \in \Omega_{2}^{*}$. Observe that there is an $\eta>0$ such that $\left(t_{1}, \widehat{t}_{1}\right) \in(0,1] \times[\eta, 1]^{N-1}$, and for every $j_{1} \in \mathbb{N}$, there is a $\widehat{k}_{1}\left(j_{1}\right) \in\left\{0,1, \ldots, 3^{j_{1}}\right\}^{N-1}$ with $3^{-j_{1}} \widehat{k}_{1}\left(j_{1}\right) \in[\eta, 1]^{N-1}$, such that

$$
\left|\widehat{t}_{1}-3^{-j_{1}} \widehat{k}_{1}\left(j_{1}\right)\right| \leq \sqrt{N-1} 3^{-j_{1}} .
$$

Lemma 5 implies that for some real $v>0$, integer $j_{1,0} \geq 0$ and every integer $j_{1} \geq j_{1,0}$ there is $k_{1}\left(j_{1}\right) \in\left\{0,1, \ldots, 2^{j_{1}}\right\}$ satisfying

$$
\left|t_{1}-2^{-j_{1}} k_{1}\left(j_{1}\right)\right| \leq 2^{-j_{1}\left(1-H_{1} \epsilon\right)+1}
$$

and

$$
\left|C_{j_{1}, k_{1}\left(j_{1}\right)}\left(3^{-j_{1}} \widehat{k}_{1}\left(j_{1}\right), \omega\right)\right| \geq v .
$$

On the other hand, applying Lemma 3 with $n=1$, together with (2.72) and (2.87), one obtains that for any $0<\theta<H_{1}$, there is a constant $c_{2,29}>0$ such that for all $j_{1} \in \mathbb{N}$,

$$
\left|C_{j_{1}, k_{1}\left(j_{1}\right)}\left(\widehat{t}_{1}, \omega\right)-C_{j_{1}, k_{1}\left(j_{1}\right)}\left(3^{-j_{1}} \widehat{k}_{1}\left(j_{1}\right), \omega\right)\right| \leq c_{2,29}\left(\frac{2}{3}\right)^{j_{1}\left(H_{1}-\theta\right)} \times 2^{j_{1} \theta} .
$$

Combining (2.89) and (2.90), we have that for all $j_{1} \geq j_{1,0}$,

$$
\begin{aligned}
\left|C_{j_{1}, k_{1}\left(j_{1}\right)}\left(\widehat{t}_{1}, \omega\right)\right| \geq & \left|C_{j_{1}, k_{1}\left(j_{1}\right)}\left(3^{-j_{1}} \widehat{k}_{1}\left(j_{1}\right), \omega\right)\right| \\
& -\left|C_{j_{1}, k_{1}\left(j_{1}\right)}\left(\widehat{t}_{1}, \omega\right)-C_{j_{1}, k_{1}\left(j_{1}\right)}\left(3^{-j_{1}} \widehat{k}_{1}\left(j_{1}\right), \omega\right)\right| \\
\geq & v-c_{2,29}\left(\frac{2}{3}\right)^{j_{1}\left(H_{1}-\theta\right)} \times 2^{j_{1} \theta} .
\end{aligned}
$$

By choosing $\theta>0$ small enough so that $\lim _{j_{1} \rightarrow \infty}\left(\frac{2}{3}\right)^{j_{1}\left(H_{1}-\theta\right)} \times 2^{j_{1} \theta}=0$, we see that (2.91) implies the existence of an integer $j_{1,1} \geq j_{1,0} \geq 0$ such that (2.86) holds for all $j_{1} \geq j_{1,1}$. 
We are now in a position to prove Theorem 3.

Proof of Theorem 3. Let $\Omega_{2}^{*}$ be the event in Lemma 6. Suppose, ad absurdum, that there is $\epsilon>0$ small enough, $t=\left(t_{1}, \widehat{t_{1}}\right) \in(0,1] \times(0,1]^{N-1}$ and $\omega \in \Omega_{2}^{*}$ such that

$$
\limsup _{|h| \rightarrow 0} \frac{\left|B^{H}(t+h, \omega)-B^{H}(t, \omega)\right|}{\sigma(t+h, t)^{1+\epsilon}}<\infty .
$$

Then, it follows from Lemma 4 that there is a constant $c_{2,25}>0$ such that for every $j_{1} \in \mathbb{N}$ and $k_{1} \in\left\{0,1, \ldots, 2^{j_{1}}\right\}$,

$$
\left|C_{j_{1}, k_{1}}\left(\widehat{t_{1}}, \omega\right)\right| \leq c_{2,25} 2^{-j_{1} \epsilon H_{1}}\left(1+\left|2^{j_{1}} t_{1}-k_{1}\right|\right)^{(1+\epsilon) H_{1}} .
$$

On the other hand, Lemma 6 implies the existence of $k_{1}\left(j_{1}\right) \in\left\{0,1, \ldots, 2^{j_{1}}\right\}$ satisfying (2.85), (2.86), and (2.92). Hence, for every integer $j_{1} \geq j_{1,1}$,

$$
\begin{aligned}
0<v / 2 & \leq c_{2,25} 2^{-j_{1} \epsilon H_{1}}\left(1+\left|2^{j_{1}} s_{1}-k_{1}\left(j_{1}\right)\right|\right)^{(1+\epsilon) H_{1}} \\
& \leq c_{2,25} 2^{-j_{1} \epsilon H_{1}}\left(1+2^{j_{1} \epsilon H_{1}+1}\right)^{(1+\epsilon) H_{1}} .
\end{aligned}
$$

Since $\epsilon>0$ can be taken arbitrarily small, one may suppose that $(1+\epsilon) H_{1}<1$. Then one obtains that $\lim _{j_{1} \rightarrow \infty} 2^{-j \epsilon H_{1}}\left(1+2^{j_{1} \epsilon H_{1}+1}\right)^{(1+\epsilon) H_{1}}=0$, which contradicts (2.93). This finishes the proof of Theorem 3 .

\section{Hausdorff Dimension of the Range, Graph, and Level Sets}

In this section, we study the Hausdorff and packing dimensions of the range $B^{H}\left([0,1]^{N}\right)=$ $\left\{B^{H}(t): t \in[0,1]^{N}\right\}$, the graph $\operatorname{Gr} B^{H}\left([0,1]^{N}\right)=\left\{\left(t, B^{H}(t)\right): t \in[0,1]^{N}\right\}$ and the level set $L_{x}=\left\{t \in(0, \infty)^{N}: B^{H}(t)=x\right\}$, where $x \in \mathbb{R}^{d}$ is fixed. We refer to Falconer [15] for the definitions and properties of Hausdorff and packing dimensions.

In order to state our theorems conveniently, we further assume

$$
0<H_{1} \leq \ldots \leq H_{N}<1 .
$$

Theorem 4. Let $B^{H}=\left\{B^{H}(t), t \in \mathbb{R}_{+}^{N}\right\}$ be an $(N, d)$-fractional Brownian sheet with Hurst index $H=\left(H_{1}, \ldots, H_{N}\right)$ satisfying (3.1). Then with probability 1 ,

$$
\operatorname{dim}_{\mathrm{H}} B^{H}\left([0,1]^{N}\right)=\min \left\{d ; \quad \sum_{j=1}^{N} \frac{1}{H_{j}}\right\}
$$

and

$$
\begin{aligned}
& \operatorname{dim}_{\mathrm{H}} \operatorname{Gr} B^{H}\left([0,1]^{N}\right)=\min \left\{\sum_{j=1}^{k} \frac{H_{k}}{H_{j}}+N-k+\left(1-H_{k}\right) d, 1 \leq k \leq N ; \sum_{j=1}^{N} \frac{1}{H_{j}}\right\} \\
& \quad=\left\{\begin{array}{l}
\sum_{j=1}^{N} \frac{1}{H_{j}} \\
\sum_{j=1}^{k} \frac{H_{k}}{H_{j}}+N-k+\left(1-H_{k}\right) d \quad \text { if } \sum_{j=1}^{N} \frac{1}{H_{j}} \leq d, \\
j-1 \frac{1}{H_{j}} \leq d<\sum_{j=1}^{k} \frac{1}{H_{j}},
\end{array}\right.
\end{aligned}
$$


where $\sum_{j=1}^{0} \frac{1}{H_{j}}:=0$.

Remark 3. When $d=1$, (3.3) coincides with the result of Ayache [3].

The second equality in (3.3) is verified by the following lemma, whose proof is elementary and is omitted. Denote

$$
\kappa:=\min \left\{\sum_{j=1}^{k} \frac{H_{k}}{H_{j}}+N-k+\left(1-H_{k}\right) d, 1 \leq k \leq N ; \sum_{j=1}^{N} \frac{1}{H_{j}}\right\} .
$$

Lemma 7. Assume (3.1) holds. We have

(i) If $d \geq \sum_{j=1}^{N} \frac{1}{H_{j}}$, then $\kappa=\sum_{j=1}^{N} \frac{1}{H_{j}}$.

(ii) If $\sum_{j=1}^{\ell-1} \frac{1}{H_{j}} \leq d<\sum_{j=1}^{\ell} \frac{1}{H_{j}}$ for some $1 \leq \ell \leq N$, then

$$
\kappa=\sum_{j=1}^{\ell} \frac{H_{\ell}}{H_{j}}+N-\ell+\left(1-H_{\ell}\right) d
$$

and $\kappa \in(N-\ell+d, N-\ell+d+1]$.

As usual, the proof of Theorem 4 is divided into proving the upper and lower bounds separately. In the following, we first show that the upper bounds in (3.2) and (3.3) follow from Theorem 1 and a covering argument.

Proof of the upper bounds in Theorem 4. For the proof of the upper bound in (3.2), we note that clearly $\operatorname{dim}_{\mathrm{H}} B^{H}\left([0,1]^{N}\right) \leq d$ a.s., so we only need to prove the following inequality:

$$
\operatorname{dim}_{\mathrm{H}} B^{H}\left([0,1]^{N}\right) \leq \sum_{j=1}^{N} \frac{1}{H_{j}} \quad \text { a.s. }
$$

For any constants $0<\gamma_{j}<\gamma_{j}^{\prime}<H_{j}(1 \leq j \leq N)$, it follows from Theorem 1 that there is a random variable $A_{1}$ of finite moments of all orders such that for all $\omega \in \Omega_{1}^{*}$,

$$
\sup _{s, t \in[0,1]^{N}} \frac{\left|B^{H}(s, \omega)-B^{H}(t, \omega)\right|}{\sum_{j=1}^{N}\left|s_{j}-t_{j}\right|^{\gamma_{j}^{\prime}}} \leq A_{1}(\omega) .
$$

Let $\omega \in \Omega_{1}^{*}$ be fixed and then suppressed. For any integer $n \geq 2$, we divide $[0,1]^{N}$ into $m_{n}$ sub-rectangles $\left\{R_{n, i}\right\}$ with sides parallel to the axes and side-lengths $n^{-1 / H_{j}}$ ( $j=$ $1, \ldots, N)$, respectively. Then

$$
m_{n} \leq c_{3,1} n^{\sum_{j=1}^{N} \frac{1}{H_{j}}} .
$$

and $B^{H}\left([0,1]^{N}\right)$ can be covered by $B^{H}\left(R_{n, i}\right)\left(1 \leq i \leq m_{n}\right)$ and, by (3.6), we see that the diameter of the image $B^{H}\left(R_{n, i}\right)$ satisfies

$$
\operatorname{diam} B^{H}\left(R_{n, i}\right) \leq c_{3,2} n^{-1+\delta},
$$


where $\delta=\max \left\{\left(H_{j}-\gamma_{j}^{\prime}\right) / H_{j}, 1 \leq j \leq N\right\}$. We choose $\gamma_{j}^{\prime} \in\left(\gamma_{j}, H_{j}\right)$ for each $j$ such that

$$
(1-\delta) \sum_{j=1}^{N} \frac{1}{\gamma_{j}}>\sum_{j=1}^{N} \frac{1}{H_{j}} .
$$

Hence, for $\gamma=\sum_{j=1}^{N} \frac{1}{\gamma_{j}}$, it follows from (3.7) and (3.8) that

$$
\sum_{i=1}^{m_{n}}\left[\operatorname{diam} B^{H}\left(R_{n, i}\right)\right]^{\gamma} \leq c_{3,3} n^{\sum_{j=1}^{N} \frac{1}{H_{j}}} n^{-(1-\delta) \gamma} \rightarrow 0
$$

as $n \rightarrow \infty$. This proves that $\operatorname{dim}_{\mathrm{H}} B^{H}\left([0,1]^{N}\right) \leq \gamma$ a.s. By letting $\gamma_{j} \uparrow H_{j}$ along rational numbers, we derive (3.5).

Now we turn to the proof of the upper bound in (3.3). We will show that there are several different ways to cover $\operatorname{Gr} B^{H}\left([0,1]^{N}\right)$, each of which leads to an upper bound for $\operatorname{dim}_{\mathrm{H}} \operatorname{Gr} B^{H}\left([0,1]^{N}\right)$.

For each fixed integer $n \geq 2$, we have

$$
\operatorname{Gr} B^{H}\left([0,1]^{N}\right) \subseteq \bigcup_{i=1}^{m_{n}} R_{n, i} \times B^{H}\left(R_{n, i}\right) .
$$

It follows from (3.8) and (3.9) that $\operatorname{Gr} B^{H}\left([0,1]^{N}\right)$ can be covered by $m_{n}$ cubes in $\mathbb{R}^{N+d}$ with side-lengths $c_{3,4} n^{-1+\delta}$ and the same argument as the above yields

$$
\operatorname{dim}_{\mathrm{H}} \mathrm{Gr} B^{H}\left([0,1]^{N}\right) \leq \sum_{j=1}^{N} \frac{1}{H_{j}} \quad \text { a.s. }
$$

We fix an integer $1 \leq k \leq N$. Observe that each $R_{n, i} \times B^{H}\left(R_{n, i}\right)$ can be covered by $\ell_{n, k}$ cubes in $\mathbb{R}^{N+d}$ of sides $n^{-\frac{1}{H_{k}}}$, where by (3.6)

$$
\ell_{n, k} \leq c_{3,5} n^{\sum_{j=k}^{N}\left(\frac{1}{H_{k}}-\frac{1}{H_{j}}\right)} \times n^{\left(\frac{1}{H_{k}}-1+\delta\right) d} .
$$

Hence, $\operatorname{Gr} B^{H}\left([0,1]^{N}\right)$ can be covered by $m_{n} \times \ell_{n, k}$ cubes in $\mathbb{R}^{N+d}$ with sides $n^{-\frac{1}{H_{k}}}$. Denote

$$
\eta_{k}=\sum_{j=1}^{k} \frac{H_{k}}{H_{j}}+N-k+\left(1-\gamma_{k}\right) d .
$$

Recall from the above that we can choose the constants $\gamma_{k}$ and $\gamma_{j}^{\prime}(1 \leq j \leq N)$ such that $1-\delta>\frac{\gamma_{k}}{H_{k}}$. Some simple calculations show that

$$
m_{n} \times \ell_{n, k} \times\left(n^{-\frac{1}{H_{k}}}\right)^{\eta_{k}} \leq c_{3,6} n^{-\left(1-\delta-\frac{\gamma_{k}}{H_{k}}\right) d} \rightarrow 0
$$

as $n \rightarrow \infty$. This implies that $\operatorname{dim}_{\mathrm{H}} \operatorname{Gr} B^{H}\left([0,1]^{N}\right) \leq \eta_{k}$ almost surely. Therefore for every $k=1, \ldots, N$,

$$
\operatorname{dim}_{\mathrm{H}} \operatorname{Gr} B^{H}\left([0,1]^{N}\right) \leq \sum_{j=1}^{k} \frac{H_{k}}{H_{j}}+N-k+\left(1-H_{k}\right) d .
$$


Combining (3.10) and (3.11) yields the upper bound in (3.3).

For proving the lower bounds in Theorem 4, we need several lemmas.

Lemma 8. For any $\varepsilon>0$, there exist positive and finite constants $c_{3,7}$ and $c_{3,8}$ such that for all $s, t \in[\varepsilon, 1]^{N}$,

$$
c_{3,7} \sum_{j=1}^{N}\left|s_{j}-t_{j}\right|^{2 H_{j}} \leq \mathbb{E}\left[\left(B_{0}^{H}(s)-B_{0}^{H}(t)\right)^{2}\right] \leq c_{3,8} \sum_{j=1}^{N}\left|s_{j}-t_{j}\right|^{2 H_{j}} .
$$

Remark 4. The upper bound in (3.12) holds for all $s, t \in[0,1]^{N}$ and $c_{3,8}$ is independent of $\varepsilon$. However, the constant $c_{3,7}$ depends on $\varepsilon$.

Proof of Lemma 8. In order to prove the upper bound in (3.12), we use (1.1) and write $\mathbb{E}\left[\left(B_{0}^{H}(s)-B_{0}^{H}(t)\right)^{2}\right]$ as

$$
\begin{aligned}
& {\left[\prod_{j=1}^{N-1} s_{j}^{2 H_{j}}+\prod_{j=1}^{N-1} t_{j}^{2 H_{j}}-2 \prod_{j=1}^{N-1} \frac{1}{2}\left(s_{j}^{2 H_{j}}+t_{j}^{2 H_{j}}-\left|s_{j}-t_{j}\right|^{2 H_{j}}\right)\right] s_{N}^{2 H_{N}}} \\
& \quad+\left[\prod_{j=1}^{N-1} t_{j}^{2 H_{j}}-\prod_{j=1}^{N-1} \frac{1}{2}\left(s_{j}^{2 H_{j}}+t_{j}^{2 H_{j}}-\left|s_{j}-t_{j}\right|^{2 H_{j}}\right)\right]\left(t_{N}^{2 H_{N}}-s_{N}^{2 H_{N}}\right) \\
& \quad+\left|t_{N}-s_{N}\right|^{2 H_{N}} \cdot \prod_{j=1}^{N-1} \frac{1}{2}\left(s_{j}^{2 H_{j}}+t_{j}^{2 H_{j}}-\left|s_{j}-t_{j}\right|^{2 H_{j}}\right) \\
& \quad:=T_{1}+T_{2}+T_{3} .
\end{aligned}
$$

It is clear that $\left|T_{2}\right| \leq c\left|t_{N}-s_{N}\right|^{2 H_{N}}$ and $T_{3} \leq\left|t_{N}-s_{N}\right|^{2 H_{N}}$ for all $s, t \in[\varepsilon, 1]^{N}$. Therefore the upper bound in (3.12) follows from (3.13) and induction on $N$.

It seems to be quite involved to apply a similar elementary method to prove the lower bound in (3.12); see Xiao and Zhang [36, pp. 213-214] for an application of this argument to a similar, but easier problem. Instead, we will proceed by making use of the stochastic integral representation (1.4). We believe that our argument below will be useful in further studying other problems such as the sharp Hölder conditions for the local times and the exact Hausdorff measure of the image and graph sets of fractional Brownian sheet $B^{H}$.

Let $Y=\left\{Y(t), t \in \mathbb{R}_{+}^{N}\right\}$ be the Gaussian random field defined by

$$
\begin{aligned}
Y(t) & =\int_{0}^{t_{1}} \cdots \int_{0}^{t_{N}} g(t, r) W(d r) \\
& =\int_{0}^{t_{1}} \cdots \int_{0}^{t_{N}} \prod_{j=1}^{N}\left(t_{j}-r_{j}\right)^{H_{j}-\frac{1}{2}} W(d r), \quad t \in \mathbb{R}_{+}^{N} .
\end{aligned}
$$

Then by the independence of the Brownian sheet on different quadrants of $\mathbb{R}^{N}$, we have $\mathbb{E}\left[\left(B_{0}^{H}(s)-B_{0}^{H}(t)\right)^{2}\right] \geq \kappa_{H}^{-2} \mathbb{E}\left[(Y(s)-Y(t))^{2}\right]$ for all $s, t \in[\varepsilon, 1]^{N}$.

For every $t \in[\varepsilon, 1]^{N}$, we decompose the rectangle $[0, t]$ into the following disjoint union:

$$
[0, t]=[0, \varepsilon]^{N} \cup \bigcup_{j=1}^{N} R\left(t_{j}\right) \cup \Delta(\varepsilon, t)
$$


where $R\left(t_{j}\right)=\left\{r \in[0,1]^{N}: 0 \leq r_{i} \leq \varepsilon\right.$, if $\left.i \neq j, \varepsilon<r_{j} \leq t_{j}\right\}$ and $\Delta(\varepsilon, t)$ can be written as a union of $2^{N}-N-1$ sub-rectangles of $[0, t]$. It follows from (3.14) and (3.15) that for every $t \in[\varepsilon, 1]^{N}$,

$$
\begin{aligned}
Y(t) & =\int_{[0, \varepsilon]^{N}} g(t, r) W(d r)+\sum_{j=1}^{N} \int_{R\left(t_{j}\right)} g(t, r) W(d r)+\int_{\Delta(\varepsilon, t)} g(t, r) W(d r) \\
& :=X(\varepsilon, t)+\sum_{j=1}^{N} Y_{j}(t)+Z(\varepsilon, t) .
\end{aligned}
$$

Since the processes $X(\varepsilon, t), Y_{j}(t)(1 \leq j \leq N)$ and $Z(\varepsilon, t)$ are defined by the stochastic integrals over disjoint sets, they are independent. Only the $Y_{j}(t)$ 's will be useful for proving the lower bound in (3.12).

Now let $s, t \in[\varepsilon, 1]^{N}$ and $1 \leq j \leq N$ be fixed. Without loss of generality, we assume $s_{j} \leq t_{j}$. Then

$$
\mathbb{E}\left[\left(Y_{j}(t)-Y_{j}(s)\right)^{2}\right]=\int_{R\left(s_{j}\right)}(g(t, r)-g(s, r))^{2} d r+\int_{R\left(s_{j}, t_{j}\right)} g^{2}(t, r) d r
$$

where $R\left(s_{j}, t_{j}\right)=\left\{r \in[0,1]^{N}: 0 \leq r_{i} \leq \varepsilon\right.$, if $\left.i \neq j, s_{j}<r_{j} \leq t_{j}\right\}$. By (3.17) and some elementary calculations we derive

$$
\begin{aligned}
\mathbb{E}\left[\left(Y_{j}(t)-Y_{j}(s)\right)^{2}\right] & \geq \int_{R\left(s_{j}, t_{j}\right)} g^{2}(t, r) d r \\
& =\int_{[0, \varepsilon]^{N-1}} \prod_{k \neq j}\left(t_{k}-r_{k}\right)^{2 H_{k}-1} \int_{s_{j}}^{t_{j}}\left(t_{j}-r_{j}\right)^{2 H_{j}-1} d r \\
& \geq c_{3,9}\left|t_{j}-s_{j}\right|^{2 H_{j}},
\end{aligned}
$$

where $c_{3,9}$ is a positive constant depending on $\varepsilon$ and $H_{k}(1 \leq k \leq N)$ only.

It follows from (3.16), (3.17), and (3.18) that for all $s, t \in[\varepsilon, 1]^{N}$,

$$
\begin{aligned}
\mathbb{E}\left[(Y(s)-Y(t))^{2}\right] & \geq \sum_{j=1}^{N} \mathbb{E}\left[\left(Y_{j}(s)-Y_{j}(t)\right)^{2}\right] \\
& \geq c_{3,9} \sum_{j=1}^{N}\left|s_{j}-t_{j}\right|^{2 H_{j}} .
\end{aligned}
$$

This proves (3.12) with $c_{3,7}=c_{3,9} \kappa_{H}^{-2}$.

Lemma 9 below is proved in Xiao and Zhang [36, p. 212] which will be used to derive a lower bound for $\operatorname{dim}_{\mathrm{H}} B^{H}\left([0,1]^{N}\right)$. Lemma 10 is needed for determining a lower bound for $\operatorname{dim}_{\mathrm{H}} \operatorname{Gr} B^{H}\left([0,1]^{N}\right)$. Both lemmas will be useful in the proof of Theorem 5 .

Lemma 9. Let $0<\alpha<1$ and $\varepsilon>0$ be given constants. Then for any constants $\delta>2 \alpha$, $M>0$ and $p>0$, there exists a positive and finite constant $c_{3,10}$, depending on $\varepsilon, \delta, p$ and $M$ only, such that for all $0<A \leq M$,

$$
\int_{\varepsilon}^{1} d s \int_{\varepsilon}^{1} \frac{1}{\left(A+|s-t|^{2 \alpha}\right)^{p}} d t \leq c_{3,10}\left(A^{-\left(p-\frac{1}{\delta}\right)}+1\right) .
$$


Lemma 10. Let $\alpha, \beta$ and $\eta$ be positive constants. For $A>0$ and $B>0$, let

$$
J:=J(A, B)=\int_{0}^{1} \frac{d t}{\left(A+t^{\alpha}\right)^{\beta}(B+t)^{\eta}} .
$$

Then there exist finite constants $c_{3,11}$ and $c_{3,12}$, depending on $\alpha, \beta, \eta$ only, such that the following hold for all reals $A, B>0$ satisfying $A^{1 / \alpha} \leq c_{3,11} B$ :

(i) If $\alpha \beta>1$, then

$$
J \leq c_{3,12} \frac{1}{A^{\beta-\alpha^{-1}} B^{\eta}}
$$

(ii) if $\alpha \beta=1$, then

$$
J \leq c_{3,12} \frac{1}{B^{\eta}} \log \left(1+B A^{-1 / \alpha}\right) ;
$$

(iii) if $0<\alpha \beta<1$ and $\alpha \beta+\eta \neq 1$, then

$$
J \leq c_{3,12}\left(\frac{1}{B^{\alpha \beta+\eta-1}}+1\right) .
$$

Proof. By a change of variable, we have

$$
J=\frac{1}{B^{\eta-1}} \int_{0}^{B^{-1}} \frac{d t}{\left(A+B^{\alpha} t^{\alpha}\right)^{\beta}(1+t)^{\eta}} .
$$

To prove (i) and (ii), we note that, if $B<1$, then we can split the integral in (3.25) so that

$$
J=\frac{1}{B^{\eta-1}} \int_{0}^{1} \frac{d t}{\left(A+B^{\alpha} t^{\alpha}\right)^{\beta}(1+t)^{\eta}}+\frac{1}{B^{\eta-1}} \int_{1}^{B^{-1}} \frac{d t}{\left(A+B^{\alpha} t^{\alpha}\right)^{\beta}(1+t)^{\eta}} .
$$

If $B \geq 1$, then $J$ is bounded by the first term in (3.26). Hence, in the following, it is sufficient to consider the case $0<B<1$.

When $\alpha \beta>1$, by using (3.26) and changing the variables again, we get

$$
\begin{aligned}
J & \leq \frac{1}{B^{\eta-1}} \int_{0}^{1} \frac{d t}{\left(A+B^{\alpha} t^{\alpha}\right)^{\beta}}+\frac{1}{B^{\eta-1}} \int_{1}^{B^{-1}} \frac{d t}{\left(A+B^{\alpha} t^{\alpha}\right)^{\beta} t^{\eta}} \\
& \leq \frac{1}{A^{\beta-\alpha^{-1} B^{\eta}}} \int_{0}^{B A^{-1 / \alpha}} \frac{d s}{\left(1+s^{\alpha}\right)^{\beta}}+\frac{1}{A^{\beta-\alpha^{-1}+\eta \alpha^{-1}}} \int_{B A^{-1 / \alpha}}^{\infty} \frac{d s}{\left(1+s^{\alpha}\right)^{\beta} s^{\eta}} \\
& \leq \frac{c}{A^{\beta-\alpha^{-1}} B^{\eta}}+\frac{1}{A^{\beta-\alpha^{-1}+\eta \alpha^{-1}}} \int_{B A^{-1 / \alpha}}^{\infty} \frac{d s}{s^{\alpha \beta+\eta}} \\
& \leq \frac{c}{A^{\beta-\alpha^{-1}} B^{\eta}}+\frac{c}{B^{\alpha \beta+\eta-1}} \\
& \leq \frac{c_{3,12}}{A^{\beta-\alpha^{-1} B^{\eta}}},
\end{aligned}
$$

where in deriving the third and the last inequalities, we have used the fact that $\alpha \beta>1$ and $B A^{-1 / \alpha} \geq c_{3,11}^{-1}$. 
When $\alpha \beta=1$, similar to (3.27), we have

$$
\begin{aligned}
J & \leq \frac{1}{B^{\eta}} \int_{0}^{B A^{-1 / \alpha}} \frac{d s}{\left(1+s^{\alpha}\right)^{\beta}}+\frac{1}{A^{\eta \alpha^{-1}}} \int_{B A^{-1 / \alpha}}^{\infty} \frac{d s}{\left(1+s^{\alpha}\right)^{\beta} s^{\eta}} \\
& \leq \frac{2}{B^{\eta}} \int_{0}^{B A^{-1 / \alpha}} \frac{d s}{1+s}+\frac{1}{A^{\eta \alpha^{-1}}} \int_{B A^{-1 / \alpha}}^{\infty} \frac{d s}{s^{1+\eta}} \\
& \leq \frac{c_{3,12}}{B^{\eta}} \log \left(1+B A^{-1 / \alpha}\right) .
\end{aligned}
$$

Hence, (3.23) holds.

Finally, we consider the case $0<\alpha \beta<1$. If we further have $\alpha \beta+\eta<1$, then it follows from (3.21) that $J \leq \int_{0}^{1} \frac{d t}{t^{\alpha \beta+\eta}}<\infty$ and (3.24) holds. So it only remains to consider the case $0<\alpha \beta<1$ and $\alpha \beta+\eta>1$. For simplicity, we assume $c_{3,12}=1$ and split the integral in (3.25) as

$$
\begin{aligned}
J & =\frac{1}{B^{\eta-1}}\left(\int_{0}^{B^{-1} A^{1 / \alpha}} \frac{d t}{\left(A+B^{\alpha} t^{\alpha}\right)^{\beta}(1+t)^{\eta}}+\int_{B^{-1} A^{1 / \alpha}}^{B^{-1}} \frac{d t}{\left(A+B^{\alpha} t^{\alpha}\right)^{\beta}(1+t)^{\eta}}\right) \\
& \leq \frac{1}{A^{\beta-\alpha^{-1} B^{\eta}}}\left(\int_{0}^{1} \frac{d s}{\left(1+s^{\alpha}\right)^{\beta}}+\int_{1}^{A^{-1 / \alpha}} \frac{d s}{\left(1+s^{\alpha}\right)^{\beta}\left(1+B^{-1} A^{1 / \alpha} s\right)^{\eta}}\right) \\
& \leq \frac{c}{A^{\beta-\alpha^{-1}} B^{\eta}}+\frac{1}{A^{\beta-\alpha^{-1} B^{\eta}}}\left(\int_{1}^{B A^{-1 / \alpha}} \frac{d s}{s^{\alpha \beta}}+\frac{1}{\left(B^{-1} A^{1 / \alpha}\right)^{\eta}} \int_{B A^{-1 / \alpha}}^{A^{-1 / \alpha}} \frac{d s}{s^{\alpha \beta+\eta}}\right) .
\end{aligned}
$$

Since $\alpha \beta+\eta>1$, we have

$$
\begin{aligned}
J & \leq \frac{c}{A^{\beta-\alpha^{-1}} B^{\eta}}+\frac{1}{A^{\beta-\alpha^{-1}} B^{\eta}} \frac{c}{\left(B A^{-\alpha^{-1}}\right)^{\alpha \beta-1}} \\
& \leq \frac{c}{A^{\beta-\alpha^{-1} B^{\eta}}}+\frac{c}{B^{\alpha \beta+\eta-1}} \\
& \leq \frac{c_{3,12}}{B^{\alpha \beta+\eta-1}},
\end{aligned}
$$

where the last inequality follows from the assumption that $0<\alpha \beta<1$ and $B A^{-1 / \alpha} \geq c_{3,11}^{-1}$. Thus, (3.24) holds and the proof of Lemma 10 is finished.

Proof of the lower bounds in Theorem 4. First we prove the lower bound in (3.2). Note that for any $\varepsilon \in(0,1), \operatorname{dim}_{\mathrm{H}} B^{H}\left([0,1]^{N}\right) \geq \operatorname{dim}_{\mathrm{H}} B^{H}\left([\varepsilon, 1]^{N}\right)$. Hence, by Frostman's theorem (see e.g., Kahane [17, Chapter 10]), it is sufficient to show that for all $0<\gamma<$ $\min \left\{d, \sum_{j=1}^{N} \frac{1}{H_{j}}\right\}$

$$
\mathcal{E}_{\gamma}=\int_{[\varepsilon, 1]^{N}} \int_{[\varepsilon, 1]^{N}} \mathbb{E}\left(\frac{1}{\left|B^{H}(s)-B^{H}(t)\right|^{\gamma}}\right) d s d t<\infty
$$

Since $0<\gamma<d$, we have $0<\mathbb{E}\left(|\Xi|^{-\gamma}\right)<\infty$, where $\Xi$ is a standard $d$-dimensional normal vector. Combining this fact with Lemma 8 , we have

$$
\mathcal{E}_{\gamma} \leq c_{3,13} \int_{\varepsilon}^{1} d s_{1} \int_{\varepsilon}^{1} d t_{1} \cdots \int_{\varepsilon}^{1} d s_{N} \int_{\varepsilon}^{1} \frac{1}{\left(\sum_{j=1}^{N}\left|s_{j}-t_{j}\right|^{2 H_{j}}\right)^{\gamma / 2}} d t_{N} .
$$


To prove the above integral is finite, we observe that for any $0<\gamma<\min \left\{d, \sum_{j=1}^{N} \frac{1}{H_{j}}\right\}$, there exists an integer $1 \leq k \leq N$ such that

$$
\sum_{j=k+1}^{N} \frac{1}{H_{j}}<\gamma \leq \sum_{j=k}^{N} \frac{1}{H_{j}}
$$

where $\sum_{j=N+1}^{N} \frac{1}{H_{j}}:=0$. In the following, we will only consider the case of $k=1$, the remaining cases are simpler because they require less steps of integration using Lemma 9.

Now assuming (3.33), we choose positive constants $\delta_{2}, \ldots, \delta_{N}$ such that $\delta_{j}>2 H_{j}$ for each $2 \leq j \leq N$ and

$$
\frac{1}{\delta_{2}}+\cdots+\frac{1}{\delta_{N}}<\frac{\gamma}{2}<\frac{1}{2 H_{1}}+\frac{1}{\delta_{2}}+\cdots+\frac{1}{\delta_{N}} .
$$

Applying Lemma 9 to (3.32) with

$$
A=\sum_{j=1}^{N-1}\left|s_{j}-t_{j}\right|^{2 H_{j}} \quad \text { and } p=\gamma / 2,
$$

we obtain from (3.32) that

$\mathcal{E}_{\gamma} \leq c_{3,14}+c_{3,14} \int_{\varepsilon}^{1} d s_{1} \int_{\varepsilon}^{1} d t_{1} \cdots \int_{\varepsilon}^{1} d s_{N-1} \int_{\varepsilon}^{1} \frac{1}{\left(\sum_{j=1}^{N-1}\left|s_{j}-t_{j}\right|^{2 H_{j}}\right)^{\gamma / 2-1 / \delta_{N}}} d t_{N-1}$.

By repeatedly using Lemma 9 to the integral in (3.35) for $N-2$ steps, we derive that

$$
\mathcal{E}_{\gamma} \leq c_{3,15}+c_{3,15} \int_{\varepsilon}^{1} d s_{1} \int_{\varepsilon}^{1} \frac{1}{\left(\left|s_{1}-t_{1}\right|^{2 H_{1}}\right)^{\gamma / 2-\left(1 / \delta_{2}+\cdots+1 / \delta_{N}\right)}} d t_{1} .
$$

Since the $\delta_{j}$ 's satisfy (3.34), we have $2 H_{1}\left[\gamma / 2-\left(1 / \delta_{2}+\cdots+1 / \delta_{N}\right)\right]<1$. Thus, the integral in the right-hand side of (3.36) is finite. This proves (3.31).

Now we prove the lower bound in (3.3). Since $\operatorname{dim}_{\mathrm{H}} \operatorname{Gr} B^{H}\left([0,1]^{N}\right) \geq$ $\operatorname{dim}_{\mathrm{H}} B^{H}\left([0,1]^{N}\right)$ always holds, we only need to consider the case when

$$
\sum_{j=1}^{k-1} \frac{1}{H_{j}} \leq d<\sum_{j=1}^{k} \frac{1}{H_{j}} \quad \text { for some } 1 \leq k \leq N .
$$

Here and in the sequel, $\sum_{j=1}^{0} \frac{1}{H_{j}}:=0$.

Let $0<\varepsilon<1$ and $0<\gamma<\sum_{j=1}^{k} \frac{H_{k}}{H_{j}}+N-k+\left(1-H_{k}\right) d$ be fixed, but arbitrary, constants. By Lemma 7, we may and will assume $\gamma \in(N-k+d, N-k+d+1)$. In order to prove $\operatorname{dim}_{\mathrm{H}} \mathrm{Gr} B^{H}\left([\varepsilon, 1]^{N}\right) \geq \gamma$ a.s., again by Frostman's theorem, it is sufficient to show

$$
\mathcal{G}_{\gamma}=\int_{[\varepsilon, 1]^{N}} \int_{[\varepsilon, 1]^{N}} \mathbb{E}\left[\frac{1}{\left(|s-t|^{2}+\left|B^{H}(s)-B^{H}(t)\right|^{2}\right)^{\gamma / 2}}\right] d s d t<\infty .
$$


Since $\gamma>d$, we note that for a standard normal vector $\Xi$ in $\mathbb{R}^{d}$ and any number $a \in \mathbb{R}$,

$$
\mathbb{E}\left[\frac{1}{\left(a^{2}+|\Xi|^{2}\right)^{\gamma / 2}}\right] \leq c_{3,16} a^{-(\gamma-d)},
$$

see e.g., Kahane [17, p. 279]. Consequently, we derive that

$$
\mathcal{G}_{\gamma} \leq c_{3,16} \int_{[\varepsilon, 1]^{N}} \int_{[\varepsilon, 1]^{N}} \frac{1}{\sigma(s, t)^{d}|s-t|^{\gamma-d}} d s d t
$$

where $\sigma^{2}(s, t)=\mathbb{E}\left[\left(B_{0}^{H}(s)-B_{0}^{H}(t)\right)^{2}\right]$. (This was obtained by Ayache [3] for $d=1$ ). By Lemma 8 and a change of variables, we have

$$
\mathcal{G}_{\gamma} \leq c_{3,17} \int_{0}^{1} d t_{N} \cdots \int_{0}^{1} \frac{1}{\left(\sum_{j=1}^{N} t_{j}^{H_{j}}\right)^{d}\left(\sum_{j=1}^{N} t_{j}\right)^{\gamma-d}} d t_{1} .
$$

In order to show the integral in (3.40) is finite, we will integrate $\left[d t_{1}\right], \ldots,\left[d t_{k}\right]$ iteratively. Furthermore, we will assume $k>1$ in (3.37). (If $k=1$, we can use (3.24) to obtain (3.44) directly).

We integrate $\left[d t_{1}\right]$ first. Since $H_{1} d>1$, we can use (3.22) of Lemma 10 with $A=\sum_{j=2}^{N} t_{j}^{H_{j}}$ and $B=\sum_{j=2}^{N} t_{j}$ to get

$$
\mathcal{G}_{\gamma} \leq c_{3,18} \int_{0}^{1} d t_{N} \cdots \int_{0}^{1} \frac{1}{\left(\sum_{j=2}^{N} t_{j}^{H_{j}}\right)^{d-1 / H_{1}}\left(\sum_{j=2}^{N} t_{j}\right)^{\gamma-d}} d t_{2} .
$$

We can repeat this procedure for integrating $d t_{2}, \ldots, d t_{k-1}$. Note that, if $d=\sum_{j=1}^{k-1} \frac{1}{H_{j}}$, then we need to use (3.23) to integrate $\left[d t_{k-1}\right]$ and obtain

$$
\mathcal{G}_{\gamma} \leq c_{3,19} \int_{0}^{1} d t_{N} \cdots \int_{0}^{1} \frac{1}{\left(\sum_{j=k}^{N} t_{j}\right)^{\gamma-d}} \log \left(1+\frac{1}{\sum_{j=k}^{N} t_{j}}\right) d t_{k}<\infty .
$$

Note that the last integral is finite since $\gamma-d<N-k+1$. On the other hand, if $d>\sum_{j=1}^{k-1} \frac{1}{H_{j}}$, then (3.22) gives

$$
\mathcal{G}_{\gamma} \leq c_{3,20} \int_{0}^{1} d t_{N} \cdots \int_{0}^{1} \frac{1}{\left(\sum_{j=k}^{N} t_{j}^{H_{j}}\right)^{d-\sum_{j=1}^{k-1} 1 / H_{j}}\left(\sum_{j=k}^{N} t_{j}\right)^{\gamma-d}} d t_{k} .
$$

We integrate $\left[d t_{k}\right]$ in (3.43) and by using (3.24), we see that

$\mathcal{G}_{\gamma} \leq c_{3,21}\left[\int_{0}^{1} d t_{N} \cdots \int_{0}^{1} \frac{1}{\left(\sum_{j=k+1}^{N} t_{j}\right)^{\gamma-d+H_{k}\left(d-\sum_{j=1}^{k-1} \frac{1}{H_{j}}\right)-1}} d t_{k+1}+1\right]<\infty$,

since $\gamma-d+H_{k}\left(d-\sum_{j=1}^{k-1} \frac{1}{H_{j}}\right)-1<N-k$. Combining (3.42) and (3.44) yields (3.38). This completes the proof of Theorem 4 . 
The following result is on the Hausdorff dimension of the level set $L_{x}=\{t \in$ $\left.(0, \infty)^{N}: B^{H}(t)=x\right\}$.

Theorem 5. Let $B^{H}=\left\{B^{H}(t), t \in \mathbb{R}_{+}^{N}\right\}$ be an $(N, d)$-fractional Brownian sheet with Hurst index $H=\left(H_{1}, \ldots, H_{N}\right)$ satisfying (3.1). If $\sum_{j=1}^{N} \frac{1}{H_{j}}<d$ then for every $x \in \mathbb{R}^{d}$, $L_{x}=\emptyset$ a.s. If $\sum_{j=1}^{N} \frac{1}{H_{j}}>d$, then for any $x \in \mathbb{R}^{d}$ and $0<\varepsilon<1$, with positive probability

$$
\begin{aligned}
\operatorname{dim}_{\mathrm{H}}\left(L_{x} \cap[\varepsilon, 1]^{N}\right) & =\min \left\{\sum_{j=1}^{k} \frac{H_{k}}{H_{j}}+N-k-H_{k} d, 1 \leq k \leq N\right\} \\
& =\sum_{j=1}^{k} \frac{H_{k}}{H_{j}}+N-k-H_{k} d, \quad \text { if } \quad \sum_{j=1}^{k-1} \frac{1}{H_{j}} \leq d<\sum_{j=1}^{k} \frac{1}{H_{j}} .
\end{aligned}
$$

Remark 5. When $\sum_{j=1}^{N} \frac{1}{H_{j}}=d$, we believe that for every $x \in \mathbb{R}^{d}, L_{x}=\emptyset$ a.s. However, we have not been able to prove this statement. In the Brownian sheet case, this was proved by Orey and Pruitt [28, Theorem 3.4]. It also follows from a result of Khoshnevisan and Shi [19].

Proof. The second equality in (3.45) follows from Lemma 7. First we prove

$$
\operatorname{dim}_{\mathrm{H}}\left(L_{x} \cap[\varepsilon, 1]^{N}\right) \leq \min \left\{\sum_{j=1}^{k} \frac{H_{k}}{H_{j}}+N-k-H_{k} d, 1 \leq k \leq N\right\} \quad \text { a.s. }
$$

and $L_{x}=\emptyset$ a.s. whenever the right-hand side of (3.46) is negative. It can be verified that the latter is equivalent to $\sum_{j=1}^{N} \frac{1}{H_{j}}<d$.

For an integer $n \geq 1$, divide the interval $[\varepsilon, 1]^{N}$ into $m_{n}=n^{\sum_{j=1}^{N} H_{j}^{-1}}$ sub-rectangles $R_{n, \ell}$ of side lengths $n^{-1 / H_{j}}(j=1, \cdots, N)$. Let $0<\delta<1$ be fixed and let $\tau_{n, \ell}$ be the lower-left vertex of $R_{n, \ell}$. Then

$$
\begin{aligned}
\mathbb{P}\left\{x \in B^{H}\left(R_{n, \ell}\right)\right\} \leq & \mathbb{P}\left\{\max _{s, t \in R_{n, \ell}}\left|B^{H}(s)-B^{H}(t)\right| \leq n^{-(1-\delta)} ; x \in B^{H}\left(R_{n, \ell}\right)\right\} \\
& +\mathbb{P}\left\{\max _{s, t \in R_{n, \ell}}\left|B^{H}(s)-B^{H}(t)\right|>n^{-(1-\delta)}\right\} \\
\leq & \mathbb{P}\left\{\left|B^{H}\left(\tau_{n, \ell}\right)-x\right| \leq n^{-(1-\delta)}\right\}+e^{-c n^{2 \delta}} \\
\leq & c_{3,22} n^{-(1-\delta) d} .
\end{aligned}
$$

In the above we have applied Lemma 2.1 in Talagrand [29] to get the second inequality. If $\sum_{j=1}^{N} \frac{1}{H_{j}}<d$, we choose $\delta>0$ such that $(1-\delta) d>\sum_{j=1}^{N} \frac{1}{H_{j}}$. Let $N_{n}$ be the number of rectangles $R_{n, \ell}$ such that $x \in B^{H}\left(R_{n, \ell}\right)$. It follows from (3.47) that

$$
\mathbb{E}\left(N_{n}\right) \leq c_{3,22} n^{\sum_{j=1}^{N} H_{j}^{-1}} n^{-(1-\delta) d} \rightarrow 0 \quad \text { as } n \rightarrow \infty
$$

Since the random variables $N_{n}$ are integer-valued, (3.48) and Fatou's lemma imply that a.s. $N_{n}=0$ for infinitely many integers $n \geq 1$. Therefore $L_{x}=\emptyset$ a.s.

Now we assume $\sum_{j=1}^{N} \frac{1}{H_{j}}>d$ and define a covering $\left\{R_{n, \ell}^{\prime}\right\}$ of $L_{x} \cap[\varepsilon, 1]^{N}$ by $R_{n, \ell}^{\prime}=R_{n, \ell}$, if $x \in B^{H}\left(R_{n, \ell}\right)$ and $R_{n, \ell}^{\prime}=\emptyset$ otherwise. For every $1 \leq k \leq N, R_{n, \ell}^{\prime}$ can be 
covered by $n^{\sum_{j=k+1}^{N}\left(H_{k}^{-1}-H_{j}^{-1}\right)}$ cubes of side length $n^{-H_{k}^{-1}}$. Thus, we can cover the level set $L_{x} \cap[\varepsilon, 1]^{N}$ by a sequence of cubes of side length $n^{-H_{k}^{-1}}$. Let $\delta \in(0,1)$ be an arbitrary constant and let

$$
\eta=\sum_{j=1}^{k} \frac{H_{k}}{H_{j}}+N-k-H_{k}(1-\delta) d .
$$

It follows from (3.47) that

$$
\begin{aligned}
& \mathbb{E}\left[\sum_{\ell=1}^{m_{n}} n^{\sum_{j=k+1}^{N}\left(H_{k}^{-1}-H_{j}^{-1}\right)}\left(n^{-H_{k}^{-1}}\right)^{\eta} \mathbf{1}_{\left\{x \in B^{H}\left(R_{n, \ell}\right)\right\}}\right] \\
& \quad \leq c_{3,23} n^{\sum_{j=1}^{N} H_{j}^{-1}+\sum_{j=k+1}^{N}\left(H_{k}^{-1}-H_{j}^{-1}\right)-\eta H_{k}^{-1}-(1-\delta) d}=c_{3,23} .
\end{aligned}
$$

Fatou's lemma implies that the $\eta$-dimensional Hausdorff measure of $L_{x} \cap[\varepsilon, 1]^{N}$ is finite a.s. and thus $\operatorname{dim}_{\mathrm{H}}\left(L_{x} \cap[\varepsilon, 1]^{N}\right) \leq \eta$ almost surely. Letting $\delta \downarrow 0$ along rational numbers, we obtain (3.46).

To prove the lower bound in (3.45), we assume $\sum_{j=1}^{k-1} \frac{1}{H_{j}} \leq d<\sum_{j=1}^{k} \frac{1}{H_{j}}$ for some $1 \leq k \leq N$. Let $\delta>0$ be a small constant such that

$$
\gamma:=\sum_{j=1}^{k} \frac{H_{k}}{H_{j}}+N-k-H_{k}(1+\delta) d>N-k .
$$

Note that, if we can prove that there is a constant $c_{3,24}>0$, independent of $\delta$ and $\gamma$, such that

$$
\mathbb{P}\left\{\operatorname{dim}_{\mathrm{H}}\left(L_{x} \cap[\varepsilon, 1]^{N}\right) \geq \gamma\right\} \geq c_{3,24},
$$

then the lower bound in (3.45) will follow by letting $\delta \downarrow 0$.

Our proof of (3.51) is based on the capacity argument due to Kahane (see [17]). Similar methods have been used by Adler [1], Testard [30], and Xiao [31].

Let $\mathcal{M}_{\gamma}^{+}$be the space of all non-negative measures on $\mathbb{R}^{N}$ with finite $\gamma$-energy. It is known (cf. Adler [1]) that $\mathcal{M}_{\gamma}^{+}$is a complete metric space under the metric

$$
\|\mu\|_{\gamma}=\int_{\mathbb{R}^{N}} \int_{\mathbb{R}^{N}} \frac{\mu(d t) \mu(d s)}{|t-s|^{\gamma}} .
$$

We define a sequence of random positive measures $\mu_{n}$ on the Borel sets $C$ of $[\varepsilon, 1]^{N}$ by

$$
\begin{aligned}
\mu_{n}(C) & =\int_{C}(2 \pi n)^{d / 2} \exp \left(-\frac{n\left|B^{H}(t)-x\right|^{2}}{2}\right) d t \\
& =\int_{C} \int_{\mathbb{R}^{d}} \exp \left(-\frac{|\xi|^{2}}{2 n}+i\left\langle\xi, B^{H}(t)-x\right\rangle\right) d \xi d t .
\end{aligned}
$$

It follows from Kahane [17], or Testard [30] that, if there are constants $c_{3,25}>$ $0, c_{3,26}>0$ such that

$$
\mathbb{E}\left(\left\|\mu_{n}\right\|\right) \geq c_{3,25}, \quad \mathbb{E}\left(\left\|\mu_{n}\right\|^{2}\right) \leq c_{3,26}
$$


and

$$
\mathbb{E}\left(\left\|\mu_{n}\right\|_{\gamma}\right)<+\infty
$$

where $\left\|\mu_{n}\right\|=\mu_{n}\left([\varepsilon, 1]^{N}\right)$, then there is a subsequence of $\left\{\mu_{n}\right\}$, say $\left\{\mu_{n_{k}}\right\}$, such that $\mu_{n_{k}} \rightarrow \mu$ in $\mathcal{M}_{\gamma}^{+}$and $\mu$ is strictly positive with probability $\geq c_{3,25}^{2} /\left(2 c_{3,26}\right)$. In this case, it follows from (3.53) that the measure $\mu$ has its support in $L_{x} \cap[\varepsilon, 1]^{N}$ almost surely. Hence, Frostman's theorem yields (3.51) with $c_{3,24}=c_{3,25}^{2} /\left(2 c_{3,26}\right)$.

It remains to verify (3.54) and (3.55). By Fubini's theorem we have

$$
\begin{aligned}
\mathbb{E}\left(\left\|\mu_{n}\right\|\right) & =\int_{[\varepsilon, 1]^{N}} \int_{\mathbb{R}^{d}} e^{-i\langle\xi, x\rangle} \exp \left(-\frac{|\xi|^{2}}{2 n}\right) \mathbb{E} \exp \left(i\left\langle\xi, B^{H}(t)\right\rangle\right) d \xi d t \\
& =\int_{[\varepsilon, 1]^{N}} \int_{\mathbb{R}^{d}} e^{-i\langle\xi, x\rangle} \exp \left(-\frac{1}{2}\left(n^{-1}+\sigma^{2}(t)\right)|\xi|^{2}\right) d \xi d t \\
& =\int_{[\varepsilon, 1]^{N}}\left(\frac{2 \pi}{n^{-1}+\sigma^{2}(t)}\right)^{d / 2} \exp \left(-\frac{|x|^{2}}{2\left(n^{-1}+\sigma^{2}(t)\right)}\right) d t \\
& \geq \int_{[\varepsilon, 1]^{N}}\left(\frac{2 \pi}{1+\sigma^{2}(t)}\right)^{d / 2} \exp \left(-\frac{|x|^{2}}{2 \sigma^{2}(t)}\right) d t:=c_{3,25} .
\end{aligned}
$$

Denote by $I_{2 d}$ the identity matrix of order $2 d, \operatorname{Cov}\left(B^{H}(s), B^{H}(t)\right)$ the covariance matrix of $\left(B^{H}(s), B^{H}(t)\right), \Gamma=n^{-1} I_{2 d}+\operatorname{Cov}\left(B^{H}(s), B^{H}(t)\right)$ and $(\xi, \eta)^{\prime}$ the transpose of the row vector $(\xi, \eta)$. Then

$$
\begin{aligned}
\mathbb{E}\left(\left\|\mu_{n}\right\|^{2}\right) & =\int_{[\varepsilon, 1]^{N}} \int_{[\varepsilon, 1]^{N}} \int_{\mathbb{R}^{d}} \int_{\mathbb{R}^{d}} e^{-i\langle\xi+\eta, x\rangle} \exp \left(-\frac{1}{2}(\xi, \eta) \Gamma(\xi, \eta)^{\prime}\right) d \xi d \eta d s d t \\
& =\int_{[\varepsilon, 1]^{N}} \int_{[\varepsilon, 1]^{N}} \frac{(2 \pi)^{d}}{\sqrt{\operatorname{det} \Gamma}} \exp \left(-\frac{1}{2}(x, x) \Gamma^{-1}(x, x)^{\prime}\right) d s d t \\
& \leq \int_{[\varepsilon, 1]^{N}} \int_{[\varepsilon, 1]^{N}} \frac{(2 \pi)^{d}}{\left[\operatorname{det} \operatorname{Cov}\left(B_{0}^{H}(s), B_{0}^{H}(t)\right)\right]^{d / 2}} d s d t .
\end{aligned}
$$

It can be proven (see Xiao and Zhang [36, p. 214]) that for all $s, t \in[\varepsilon, 1]^{N}$,

$$
\begin{aligned}
\operatorname{det} \operatorname{Cov}\left(B_{0}^{H}(s), B_{0}^{H}(t)\right) & =\prod_{j=1}^{N} s_{j}^{2 H_{j}} t_{j}^{2 H_{j}}-\prod_{j=1}^{N} \frac{1}{4}\left(s_{j}^{2 H_{j}}+t_{j}^{2 H_{j}}-\left|s_{j}-t_{j}\right|^{2 H_{j}}\right) \\
& \geq c_{3,27} \sum_{j=1}^{N}\left|s_{j}-t_{j}\right|^{2 H_{j}} .
\end{aligned}
$$

Combining (3.57), (3.58) and applying Lemma 9 repeatedly, we obtain

$$
\mathbb{E}\left(\left\|\mu_{n}\right\|^{2}\right) \leq c_{3,28} \int_{[\varepsilon, 1]^{N}} \int_{[\varepsilon, 1]^{N}} \frac{1}{\left[\sum_{j=1}^{N}\left|s_{j}-t_{j}\right|^{2 H_{j}}\right]^{d / 2}} d s d t:=c_{3,26}<\infty .
$$

In the above, we have omitted the proof of $c_{3,26}<\infty$ since it is very similar to (3.32)-(3.36) in the proof of Theorem 4. Therefore we have shown (3.54) holds. 
Similar to (3.57), we have

$$
\begin{aligned}
& \mathbb{E}\left(\left\|\mu_{n}\right\|_{\gamma}\right)=\int_{[\varepsilon, 1]^{N}} \int_{[\varepsilon, 1]^{N}} \frac{d s d t}{|s-t|^{\gamma}} \int_{\mathbb{R}^{d}} \int_{\mathbb{R}^{d}} e^{-i\langle\xi+\eta, x\rangle} \exp \left(-\frac{1}{2}(\xi, \eta) \Gamma(\xi, \eta)^{\prime}\right) d \xi d \eta \\
& \leq c_{3,29} \int_{[\varepsilon, 1]^{N}} \int_{[\varepsilon, 1]^{N}} \frac{1}{\left(\sum_{j=1}^{N}\left|s_{j}-t_{j}\right|\right)^{\gamma}\left(\sum_{j=1}^{N}\left|s_{j}-t_{j}\right|^{2 H_{j}}\right)^{d / 2}} d s d t \\
& \quad \leq c_{3,30} \int_{0}^{1} d t_{N} \cdots \int_{0}^{1} \frac{1}{\left(\sum_{j=1}^{N} t_{j}^{H_{j}}\right)^{d}\left(\sum_{j=1}^{N} t_{j}\right)^{\gamma}} d t_{1},
\end{aligned}
$$

where the two inequalities follow from (3.58) and a change of variables. Note that the last integral in (3.60) is similar to (3.40). By using Lemma 10 in the same way as in the proof of (3.41)-(3.44), we see that for any $\gamma$ defined in (3.50), $\mathbb{E}\left(\left\|\mu_{n}\right\|_{\gamma}\right)<+\infty$. This proves (3.55) and hence Theorem 5.

By using the relationships among the Hausdorff dimension, packing dimension and the box dimension (see Falconer [15]), Theorems 4 and 5 and their proofs of the upper bounds, we derive the following analogous result on the packing dimensions of $B^{H}\left([0,1]^{N}\right)$, $\operatorname{Gr} B^{H}\left([0,1]^{N}\right)$ and $L_{x}$.

Theorem 6. Let $B^{H}=\left\{B^{H}(t), t \in \mathbb{R}_{+}^{N}\right\}$ be an $(N, d)$-fractional Brownian sheet with Hurst index $H=\left(H_{1}, \ldots, H_{N}\right)$ satisfying (3.1). Then with probability 1 ,

$$
\begin{aligned}
\operatorname{dim}_{\mathrm{P}} B^{H}\left([0,1]^{N}\right) & =\min \left\{d ; \quad \sum_{j=1}^{N} \frac{1}{H_{j}}\right\}, \\
\operatorname{dim}_{\mathrm{P}} \mathrm{Gr} B^{H}\left([0,1]^{N}\right) & =\min \left\{\sum_{j=1}^{k} \frac{H_{k}}{H_{j}}+N-k+\left(1-H_{k}\right) d, 1 \leq k \leq N ; \sum_{j=1}^{N} \frac{1}{H_{j}}\right\} .
\end{aligned}
$$

If $\sum_{j=1}^{N} \frac{1}{H_{j}}>d$, then for any $x \in \mathbb{R}^{d}$ and $0<\varepsilon<1$,

$$
\operatorname{dim}_{\mathrm{P}}\left(L_{x} \cap[\varepsilon, 1]^{N}\right)=\min \left\{\sum_{j=1}^{k} \frac{H_{k}}{H_{j}}+N-k-H_{k} d, 1 \leq k \leq N\right\}
$$

with positive probability.

Remark 6. In light of Theorems 4, 5, and 6, it would be interesting to determine the exact Hausdorff and packing measure functions for $B^{H}\left([0,1]^{N}\right), \operatorname{Gr} B^{H}\left([0,1]^{N}\right)$ and the level set $L_{x}$. In the special case of the Brownian sheet, the Hausdorff measure of the range and graph were evaluated by Ehm [13]. However, his method relies crucially on the independent increment property of the Brownian sheet and is not applicable to the fractional Brownian sheet $B^{H}$ in general. Moreover, no packing measure results have been proven even for random sets determined by the ordinary Brownian sheet.

Related to these problems, we mention that the Hausdorff measure functions for the range and graph of an $(N, d)$-fractional Brownian motion $X$ have been obtained by Talagrand [29] and Xiao [33, 34]; and the exact packing measure functions for $X\left([0,1]^{N}\right)$ have been studied by Xiao [32, 35]. Their methods are useful for studying the fractional Brownian sheet $B^{H}$ as well. 
Remark 7. By examining the proofs, we see that Theorems 4, 5, and 6 hold for all Gaussian random fields satisfying (3.12) and (3.58), including certain anistropic Gaussian random fields with stationary increments (see e.g., Kôno [20], Bonami and Estrade [9, Example 3]).

\section{Acknowledgments}

We thank the referee for his/her careful reading of our article and for his/her thoughtful comments that have led to several improvements of the manuscript.

\section{References}

[1] Adler, R. J. (1981). The Geometry of Random Fields, John Wiley \& Sons, New York.

[2] Albin, J. M. P. (1994). On the upper and lower classes for a stationary Gaussian stochastic process, Ann. Probab. 22, 77-93.

[3] Ayache, A. (2004). Hausdorff dimension of the graph of the fractional Brownian sheet, Rev. Mat. Iberoamericana 20, 395-412.

[4] Ayache, A. and Taqqu, M. S. (2003). Rate optimality of wavelet series approximations of fractional Brownian sheet, J. Fourier Anal. Appl. 9(5), 451-471.

[5] Ayache, A., Jaffard, S., and Taqqu, M. S. (2004). Multifractional processes with a most general random Hölder exponent, preprint.

[6] Belinski, E. and Linde, W. (2002). Small ball probabilities of fractional Brownian sheets via fractional integration operators, J. Theoret. Probab. 15, 589-612.

[7] Benson, D. A., Meerschaert, M. M., and Baeumer, B. (2004). Aquifer operator-scaling and the effect on solute mixing and dispersion, preprint.

[8] Billingsley, P. (1968). Convergence of Probability Measures, John Wiley \& Sons, New York.

[9] Bonami, A. and Estrade, A. (2003). Anisotropic analysis of some Gaussian models, J. Fourier Anal. Appl. 9(3), 215-236.

[10] Duncan, T.E., Maslowski, B., and Pasik-Duncan, B. (2002). Fractional Brownian motion and stochastic equations in Hilbert spaces, Stoch. Dyn. 2, 225-250.

[11] Dunker, T. (2000). Estimates for the small ball probabilities of the fractional Brownian sheet, J. Theoret. Probab. 13, 357-382.

[12] Dzhaparidze, K. and van Zanten, H. (2003). Optimality of an explicit series expansion of the fractional Browinan sheet, Statist. Probab. Lett., to appear.

[13] Ehm, W. (1981). Sample function properties of multi-parameter stable processes, Z. Wahrsch. verw Gebiete 56, 195-228.

[14] Eisenbaum, N. and Khoshnevisan, D. (2002). On the most visited sites of symmetric Markov processes, Stoch. Process. Appl. 101, 241-256.

[15] Falconer, K. J. (1990). Fractal Geometry - Mathematical Foundations And Applications, John Wiley \& Sons, Chichester.

[16] Hu, Y., Øksendal, B., and Zhang, T. (2000). Stochastic partial differential equations driven by multiparameter fractional white noise, Stochastic Processes, Physics and Geometry: New Interplays, II, (Leipzig, 1999), 327-337, Amer. Math. Soc., Providence, RI.

[17] Kahane, J.-P. (1985). Some Random Series of Functions, 2nd ed, Cambridge University Press, Cambridge, UK.

[18] Kamont, A. (1996). On the fractional anisotropic Wiener field, Probab. Math. Statist. 16, 85-98.

[19] Khoshnevisan, D. and Shi, Z. (1999). Brownian sheet and capacity, Ann. Probab. 27, 1135-1159.

[20] Kôno, N. (1975). Asymptotic behavior of sample functions of Gaussian random fields, J. Math. Kyoto Univ. 15, 671-707. 
[21] Kühn, T. and Linde, W. (2002). Optimal series representation of fractional Brownian sheet, Bernoulli 8 , 669-696.

[22] Kwapień, S. and Woyczyński, N. A. (1992). Random Series and Stochastic Integrals: Single and Multiple, Birkhäuser, Boston.

[23] Lemarié, P.-G. and Meyer, Y. (1986). Ondelettes et bases hilbertiennes, Rev. Mat. Iberoamericana 2, 1-18.

[24] Meyer, Y. (1992). Wavelets and Operators, Cambridge University Press, Cambridge, UK.

[25] Mason, D. M. and Shi, Z. (2001). Small deviations for some multi-parameter Gaussian processes, J. Theoret. Probab. 14, 213-239.

[26] Øksendal, B. and Zhang, T. (2000). Multiparameter fractional Brownian motion and quasi-linear stochastic partial differential equations, Stochastics and Stochastics Reports 71, 141-163.

[27] Orey, S. (1972). Growth rate of certain Gaussian processes, in Proceedings of the Sixth Berkeley Symposium on Mathematical Statistics and Probability, (University California, Berkeley, CA, 1970/1971), Vol. II, 443 451, University California Press, Berkeley.

[28] Orey, S. and Pruitt, W.E. (1973). Sample functions of the $N$-parameter Wiener process, Ann. Probab. 1, 138-163.

[29] Talagrand, M. (1995). Hausdorff measure of trajectories of multiparameter fractional Brownian motion, Ann. Probab. 23, 767-775.

[30] Testard, F. (1986). Polarité, points multiples et géométrie de certain processus gaussiens, Publ. du Laboratoire de Statistique et Probabilités de l'U.P.S. Toulouse, 01-86.

[31] Xiao, Y. (1995). Dimension results for Gaussian vector fields and index- $\alpha$ stable fields, Ann. Probab. 23, 273-291.

[32] Xiao, Y. (1996). Packing measure of the sample paths of fractional Brownian motion, Trans. Amer. Math. Soc. 348, 3193-3213.

[33] Xiao, Y. (1997a). Hölder conditions for the local times and the Hausdorff measure of the level sets of Gaussian random fields, Probab. Theory Related Fields 109, 129-157.

[34] Xiao, Y. (1997b). Hausdorff measure of the graph of fractional Brownian motion, Math. Proc. Camb. Philo. Soc. 122, 565-576.

[35] Xiao, Y. (2003). The packing measure of the trajectories of multiparameter fractional Brownian motion, Math. Proc. Camb. Philo. Soc. 135, 349-375.

[36] Xiao, Y. and Zhang, T. (2002). Local times of fractional Brownian sheets, Probab. Theory Related Fields 124, 204-226.

Received August 10, 2004

Revision received December 03, 2004

U.M.R CNRS 8524, Laboratoire Paul Painleve, Bat.M2, Universite Lille 1, 59655 Villeneuve d'Ascq Cedex, France and

U.M.R CNRS 8020 CLAREE,

IAE de Lille

104, Avenue du Peuple Belge, 59043 Lille Cedex, France

e-mail: Antoine.Ayache@math.univ-lille1.fr

Department of Statistics and Probability, A-413 Wells Hall, Michigan State University, East Lansing, MI 48823 e-mail: xiao@stt.msu.edu 